\title{
Using coordinated observations in polarized white light and Faraday rotation to probe the spatial position and magnetic field of an interplanetary sheath
}

Article

Published Version

Xiong, M., Davies, J. A., Feng, X., Owens, M., Harrison, R. A., Davis, C. J. and Liu, Y. D. (2013) Using coordinated observations in polarized white light and Faraday rotation to probe the spatial position and magnetic field of an interplanetary sheath. The Astrophysical Journal, 777 (1). 32. ISSN 0004-637X doi: https://doi.org/10.1088/0004$637 \mathrm{X} / 777 / 1 / 32$ Available at https://centaur.reading.ac.uk/36094/

It is advisable to refer to the publisher's version if you intend to cite from the work. See Guidance on citing.

To link to this article DOI: http://dx.doi.org/10.1088/0004-637X/777/1/32

Publisher: IOP Publishing

All outputs in CentAUR are protected by Intellectual Property Rights law, including copyright law. Copyright and IPR is retained by the creators or other copyright holders. Terms and conditions for use of this material are defined in the End User Agreement. 


\section{www.reading.ac.uk/centaur}

\section{CentAUR}

Central Archive at the University of Reading

Reading's research outputs online 


\title{
USING COORDINATED OBSERVATIONS IN POLARIZED WHITE LIGHT AND FARADAY ROTATION TO PROBE THE SPATIAL POSITION AND MAGNETIC FIELD OF AN INTERPLANETARY SHEATH
}

\author{
Ming Xiong ${ }^{1,2}$, Jackie A. Davies ${ }^{3}$, Xueshang Feng $^{1}$, Mathew J. Owens ${ }^{4}$, \\ Richard A. Harrison ${ }^{3}$, Chris J. Davis ${ }^{4}$, AND Ying D. LiU ${ }^{1}$ \\ ${ }^{1}$ State Key Laboratory of Space Weather, Center for Space Science and Applied Research, \\ Chinese Academy of Sciences, Beijing, China; mxiong@ @pacweather.ac.cn \\ ${ }^{2}$ Science and Technology on Aerospace Flight Dynamics Laboratory, Beijing Aerospace Control Center, Beijing, China \\ ${ }^{3}$ Rutherford-Appleton Laboratory (RAL) Space, Harwell Oxford, UK \\ ${ }^{4}$ Reading University, Reading, UK \\ Received 2013 February 2; accepted 2013 August 13; published 2013 October 11
}

\begin{abstract}
Coronal mass ejections (CMEs) can be continuously tracked through a large portion of the inner heliosphere by direct imaging in visible and radio wavebands. White light (WL) signatures of solar wind transients, such as CMEs, result from Thomson scattering of sunlight by free electrons and therefore depend on both viewing geometry and electron density. The Faraday rotation (FR) of radio waves from extragalactic pulsars and quasars, which arises due to the presence of such solar wind features, depends on the line-of-sight magnetic field component $B_{\|}$and the electron density. To understand coordinated WL and FR observations of CMEs, we perform forward magnetohydrodynamic modeling of an Earth-directed shock and synthesize the signatures that would be remotely sensed at a number of widely distributed vantage points in the inner heliosphere. Removal of the background solar wind contribution reveals the shock-associated enhancements in WL and FR. While the efficiency of Thomson scattering depends on scattering angle, WL radiance $I$ decreases with heliocentric distance $r$ roughly according to the expression $I \propto r^{-3}$. The sheath region downstream of the Earth-directed shock is well viewed from the L4 and L5 Lagrangian points, demonstrating the benefits of these points in terms of space weather forecasting. The spatial position of the main scattering site $\mathbf{r}_{\text {sheath }}$ and the mass of plasma at that position $M_{\text {sheath }}$ can be inferred from the polarization of the shock-associated enhancement in WL radiance. From the FR measurements, the local $B_{\| \text {sheath }}$ at $\mathbf{r}_{\text {sheath }}$ can then be estimated. Simultaneous observations in polarized WL and FR can not only be used to detect CMEs, but also to diagnose their plasma and magnetic field properties.
\end{abstract}

Key words: methods: numerical - shock waves - solar-terrestrial relations - solar wind - Sun: coronal mass ejections (CMEs) - Sun: heliosphere

Online-only material: color figures

\section{INTRODUCTION}

\subsection{The Inner Heliosphere}

The inner heliosphere is permeated with a magnetized solar wind from the Sun. At solar minimum, the solar wind is inherently bimodal (McComas et al. 2000), with slow flow tending to emanate from near the ecliptic and fast flow tending to emanate at higher latitudes. Several large-scale structures, which pervade interplanetary space, are associated with the "ambient" solar wind: (1) a spiraling interplanetary magnetic field (the Parker spiral) that forms as a result of solar rotation (Parker 1958), (2) corotating interacting regions (CIRs) that are formed at the interface between a preceding slow solar wind stream and a following fast solar wind stream (Gosling \& Pizzo 1999), and (3) the heliospheric current sheet typically embedded in the heliospheric plasma sheet (Winterhalter et al. 1994; Crooker et al. 2004).

The background solar wind flow is frequently disturbed by coronal mass ejections (CMEs), large-scale expulsions of plasma and magnetic field from the solar atmosphere. CMEs typically expand during their propagation because the total solar wind pressure decreases with heliocentric distance (Démoulin \& Dasso 2009; Gulisano et al. 2010). The expansion speed of a CME depends on its spatial size, translation speed, and heliocentric distance, as well as on pre-existing solar wind conditions (Nakwacki et al. 2011; Gulisano et al. 2012). A number of popular models describe the motion of a CME as governed by two forces: a propelling Lorentz force (Chen 1989, 1996; Chen et al. 2006) and an aerodynamic drag force (Cargill et al. 1996; Vršnak \& Gopalswamy 2002; Cargill 2004). According to these models, the drag force gradually becomes dominant in interplanetary space and the CME speed finally adjusts to the ambient solar wind speed. The equalization of the $\mathrm{CME}$ and solar wind speed occurs at very different heliospheric distances, from below 30 solar radii to beyond $1 \mathrm{AU}$, depending on the characteristics of the CME and the solar wind (Temmer et al. 2011). A CME can undergo significant, nonlinear, and irreversible evolution during its propagation as it interacts with the ambient solar wind and other CMEs (e.g., Burlaga et al. 2002; Démoulin 2010). Coronagraphic observations show that CME morphology is distorted rapidly and significantly in a structured solar wind (e.g., Savani et al. 2010, 2012; Feng et al. 2012a). Such a distortion occurs over a relatively short heliocentric distance. Interactions between multiple CMEs have been revealed by in situ observations (e.g., Burlaga et al. 1987; Wang et al. 2003; Steed et al. 2011; Möstl et al. 2012), radio burst observations (e.g., Gopalswamy et al. 2001; Oliveros et al. 2012), white light (WL) imaging (e.g., Harrison et al. 2012; Liu et al. 2012; Lugaz et al. 2012; Temmer et al. 2012; Shen et al. 2012a; Bemporad et al. 2012), and numerical magnetohydrodynamic (MHD) simulations (e.g., Lugaz et al. 2005; Xiong et al. 2007, 2009; Shen et al. 2012b). 
CMEs cause phenomena on Earth such as geomagnetic storms and solar energetic particles that can result in major space weather effects (Gopalswamy 2006; Webb \& Howard 2012). Traditionally, a CME has been defined in terms of a threepart structure involving a bright sheath, a dark cavity, and a bright filament. It is now accepted that the cavity component is an escaping magnetic flux rope that drives the CME (e.g., Rouillard et al. 2009a; DeForest et al. 2011). A high-speed flux rope can drive a fast shock ahead of itself that is much wider in angular extent than the flux rope itself. The region between the shock front and the flux rope is defined as a sheath. Within the sheath, (1) magnetic field lines are draped and compressed and (2) a plasma flow is deviated, compressed, and turbulent (e.g., Gosling \& McComas 1987; Owens et al. 2005; Liu et al. 2008). Precursor southward magnetic fields ahead of CMEs are generally compressed, making them particularly geoeffective (Tsurutani et al. 1992; Gonzalez et al. 1999). The magnetic fields in the sheath and in the flux rope can be equally important in driving major geomagnetic storms (Tsurutani et al. 1988, 1992; Szajko et al. 2013). In so-called two-dip storms, it is often the case that the first dip in the Dst index is produced by the upstream sheath and the second is produced by the driving flux rope (Echer et al. 2004; Zhang et al. 2008; Möstl et al. 2012).

\subsection{Heliospheric White Light Observations}

Heliospheric imagers (HIs) detect WL that has been Thomson scattered from free electrons. For resolved objects, such as CMEs, the power detected by an individual pixel depends linearly on the solid angle subtended by that pixel $(\delta \omega)$ and the area subtended by the corresponding aperture $(\delta A)$ and is proportional to the radiance (measured in $\mathrm{W} \mathrm{m} \mathrm{m}^{-2} \mathrm{Sr}^{-1}$ ). The light from unresolved objects, such as stars, which are much narrower in angular extent, tends to fall within individual pixels. For a resolved heliospheric electron density feature, such as a CME, a single pixel provides a measure of its radiance (surface brightness), while summing contributions from all pixels over the entire extent of the feature provides a measure of its intensity (total brightness). The intensity is an integral of the radiance over the apparent feature size. Therefore, the feature's intensity determines its detectability, be it resolved or unresolved (Howard \& DeForest 2012).

The background zodiacal and stellar signals detected by HIs are much more intense than the signal due to Thomson scattering from plasma features such as CMEs (Leinert \& Pitz 1989). Fortunately, using an image-differencing technique, the much more stable background radiance can be removed, such that the more transient Thomson scattering signal can be extracted. From such processed Thomson scattering images, the sunlight-irradiated CMEs can be easily identified and tracked. According to theory, the heliospheric Thomson scattering radiance is governed by the Thomson scattering geometry factors and electron number density (Vourlidas \& Howard 2006; Howard \& Tappin 2009; Howard \& DeForest 2012; Xiong et al. 2013). The CME detectability in WL is actually more limited by perspective and field-of-view (FOV) effects than by location relative to the Thomson scattering sphere (Howard \& DeForest 2012).

Heliospheric imaging from two vantage points, both off the Sun-Earth line, was made possible by the HIs on board the Solar TErrestrial RElations Observatory (STEREO; Eyles et al. 2009). With the STEREO/SECCHI package, a CME can be imaged from its nascent stage in the inner corona all the way out to $1 \mathrm{AU}$ and beyond (e.g., Harrison et al. 2008; Davies et al. 2009; Davis et al. 2009; Liu et al. 2010; DeForest et al. 2011; Liu et al. 2013). In particular, images from STEREO/HI-2 have revealed detailed spatial structures within interplanetary CMEs, including leading-edge pileup, interior cavities, filamentary structure, and rear cusps (DeForest et al. 2011). Comparison with in situ observations has revealed that the leading-edge pileup of solar wind material, which is evident as a bright arc in WL imaging, corresponds to the sheath region. However, the interpretation of the leading edge of the radiance pattern, especially at larger elongations, is fraught with ambiguity (e.g., Howard \& Tappin 2009; Xiong et al. 2013). The elongation $\varepsilon$ is defined as the angle between the Sun-observer line and a line of sight (LOS). Because a CME occupies a significant three-dimensional (3D) volume, different parts of the CME will contribute to the radiance pattern imaged by observers situated at different heliocentric longitudes (Xiong et al. 2013). Even for an observer at a fixed longitude, a different part of the CME will contribute to the imaged radiance at any given time (Xiong et al. 2013). Various techniques have been developed that enable the spatial locations and propagation directions of CMEs to be inferred, based on fitting their moving radiance patterns (e.g., Sheeley et al. 2008; Rouillard et al. 2008; Thernisien et al. 2009; Liu et al. 2010; Lugaz et al. 2010; Möstl et al. 2011; Davies et al. 2012). However, the determination of interplanetary CME kinematics, and propagation direction in particular, is somewhat ambiguous (Howard \& Tappin 2009; Davis et al. 2010; Davies et al. 2012; Howard \& DeForest 2012; Xiong et al. 2013; Lugaz \& Kintner 2013).

\subsection{Faraday Rotation Measurement}

Faraday rotation (FR) is the rotation of the plane of polarization of an incident electromagnetic wave as it passes through a magnetized ionic medium. FR observations of linearly polarized radio sources can be used to estimate the magnetic field in the corona and interplanetary space (e.g., Levy et al. 1969; Bird et al. 1980; Sakurai \& Spangler 1994; Liu et al. 2007; Jensen 2007; Jensen \& Russell 2008; You et al. 2012; Jensen et al. 2013). The FR measurement of a radio signal corresponds to the path integral of the product of electron density $n$ and the projection of the magnetic field along the LOS, $B_{\|}$. The first FR experiment was conducted in 1968 by Levy et al. (1969), when solar plasma occulted the radio down-link from the Pioneer 6 spacecraft. As well as man-made radio sources, FR experiments can also exploit natural radio sources such as pulsars and quasars. The first FR experiments of this type were conducted by Bird et al. (1980) during the solar occultation of a pulsar. In terms of their locations on a sky map, many pulsars and quasars lie in the vicinity of the Sun. Therefore, simultaneous FR measurements along multiple beams can be used to map the inner heliosphere with reasonable spatial resolution.

Additional observations, for example in WL, would generally be necessary to confirm whether an FR transient was indeed caused by a CME. For instance, the first FR event, reported by Levy et al. (1969), could not be attributed unambiguously to the presence of any particular solar wind structure. The FR signatures exhibited a $\mathrm{W}$-shaped profile over a time period of two to three hours, with rotation angles of up to $40^{\circ}$ relative to the quiescent baseline. Woo (1997) interpreted the FR signature as the result of a coronal streamer stalk of angular size $1^{\circ}-2^{\circ}$, whereas Pätzold \& Bird (1998) argued that it was caused by the passage of a series of CMEs. However, by comparing observations from the Solwind coronagraph and measurements 
of Helios down-link radio signals, Bird et al. (1985) were able to identify the signatures of five CMEs simultaneously in WL and FR. Moreover, the electron density derived from WL imaging can be used to infer magnetic field magnitudes from FR measurements.

The heliospheric magnetic field can be remotely probed in FR using low-frequency radio interferometers such as the Murchison Widefield Array (MWA; Lonsdale, C. J., et al. 2009), the LOw Frequency ARray (LOFAR; de Vos et al. 2009), and the Very Large Array (VLA; Thompson et al. 1980). A disturbance of the background solar wind by CMEs will cause the observed FR signatures to become variable (e.g., Levy et al. 1969; Bird et al. 1985; Jensen \& Russell 2008). A change in either the electron density $(\delta n)$ or the LOS magnetic field component $\left(\delta B_{\|}\right)$or indeed both will contribute to the rotation in the plane of polarization of the radio signal by $\delta \Omega_{\mathrm{RM}}$. Interplanetary magnetic clouds (MCs) in particular, which have a magnetic flux rope configuration (Burlaga et al. 1981; Klein \& Burlaga 1982; Lepping et al. 1990), can be identified from WL images (Rouillard et al. 2009a; DeForest et al. 2011) and are expected to be easily identifiable in FR measurements. Moreover, $\delta n$ and $|\delta \mathbf{B}|$ are often enhanced simultaneously within the sheath ahead of a fast MC. The FR due to an MC-driven sheath can be comparable to that due to the MC itself (Jensen et al. 2010). It is expected that the orientation and helicity of an MC can be determined unambiguously from multi-beam FR measurements (Liu et al. 2007; Jensen 2007; Jensen et al. 2010). In contrast, the in situ detection of magnetic flux ropes can be significantly hindered by the location of the observing spacecraft (e.g., Hu \& Sonnerup 2002; Möstl et al. 2012; Démoulin et al. 2013). FR imaging can be used to provide the magnetic orientation of a fast MC and indeed its preceding sheath, prior to its arrival at Earth, which is crucial for predicting potential space weather effects on Earth.

\subsection{Forward Magnetohydrodynamic Modeling}

Forward modeling of WL and FR signatures is proving extremely useful for inferring the in situ properties of interplanetary CMEs from remote sensing data. Sophisticated numerical MHD models of the inner heliosphere (e.g., Groth et al. 2000; Lugaz et al. 2005; Hayashi 2005; Xiong et al. 2006a; Li et al. 2006; Wu et al. 2006; Li \& Li 2008; Odstrčil \& Pizzo 2009; Shen et al. 2012b) can serve as a digital laboratory to enable the synthesis of a variety of observable remote sensing signatures. In this paper, we perform a numerical MHD simulation of an interplanetary shock in the ecliptic, from which we synthesize the signatures of that feature that would be remotely sensed at visible and radio wavelengths. Details of the MHD model, and the formulae required to synthesize the remote sensing observations, are given in Section 2. The resulting synthesized remote sensing signatures of the sheath, which would be observed from vantage points at 0.5 and $1 \mathrm{AU}$, are described and compared in Section 3. In Section 4, we discuss the radiance patterns that are observed in the synthesized WL and FR sky maps. In Section 5, we explore the role that the vantage point of the observer plays in the "observability" of such WL and FR features. CME detection in the presence of background noise and the heliospheric imaging of more complex interplanetary phenomena are discussed in Section 6. The potentially important role that forward modeling can play in our understanding of coordinated WL and FR observations is summarized in Section 7 .

\section{METHOD}

Forward MHD modeling can self-consistently establish links between interplanetary dynamics and the resulting observable signatures. A complete flow chart of forward modeling is illustrated in Figure 8 of Xiong et al. (2011). The traveling fast shock studied by Xiong et al. (2013) is revisited here. Our methodology consists of three general steps: (1) forward modeling of the shock using the numerical Inner-Heliosphere MHD (IH-MHD) model (Xiong et al. 2006a, 2013), (2) calculation of its Thomson-scattered WL signature, in Section 2.1, and (3) calculation of its FR signature, in Section 2.2. A characterization of the IH-MHD model, the background solar wind conditions, and the initial shock injection is summarized in Tables 1, 2, and 3 of Xiong et al. (2013), respectively. The simulated electron density $n$ and magnetic field $\mathbf{B}$ are used to generate synthetic WL and FR images, which enable us to explore the WL and FR signatures of an interplanetary sheath.

A plasma parcel emitted from the Sun would be observed, at the same elongation and the same Thomson scattering angle, first by an observer situated at a radial distance of $0.5 \mathrm{AU}$ from the Sun center and subsequently by an observer at $1 \mathrm{AU}$ (Figure 1(a)). Such a configuration was discussed qualitatively by Jackson et al. (2010) and is analyzed quantitatively in Section 3 of this paper. Observations from STEREO/HI suggest that a traveling sheath can be approximated as an expanding bubble (e.g., Howard \& Tappin 2009; Lugaz et al. 2010; Davies et al. 2012; Möstl \& Davies 2013). In situ observations indicate that CMEs undergo self-similar expansion, as the speed profiles within CMEs themselves tend to be a linear function of time (e.g., Farrugia et al. 1993; Gulisano et al. 2012). In the schematic Figures 1(b)-(d), the sheath region following an Earth-directed interplanetary shock is represented as a self-similarly expanding bubble. The sheath can look quite different when viewed from different heliocentric distances (Figures 1 (b) and (c)) and/or different heliospheric longitudes (Figures 1 (b) and (d)).

\subsection{Thomson Scattering WL Formulae}

A small parcel of free electrons that is illuminated by a known intensity of incident sunlight (measured in $\mathrm{W} \mathrm{m}^{-2}$ ) will scatter a certain amount of power per unit solid angle (measured in $\mathrm{W} \mathrm{Sr}^{-1}$ ). The effect of the Thomson scattering geometry can be characterized by the so-called scattering angle $\chi$, as depicted in Figure 1 of Xiong et al. (2013). Scattering can be backward $\left(\chi<90^{\circ}\right)$, perpendicular $\left(\chi=90^{\circ}\right)$, and forward $\left(\chi>90^{\circ}\right)$. All photons that are scattered into an optical cone defined by the point spread function of an individual pixel will be attributed to that pixel (Figure 1(b), Xiong et al. 2013). The classic principles of WL Thomson scattering, as applied to coronagraphic observations (Billings 1966), have been adapted to heliospheric imaging (Vourlidas \& Howard 2006; Howard \& Tappin 2009; Jackson et al. 2010; Howard $\&$ DeForest 2012; Xiong et al. 2013). The transverse electric field oscillation $\delta \mathbf{E}$ of the Thomson-scattered radiance, which is inherently a continuum, can be considered in terms of its two orthogonal components, a tangential component $\delta \mathbf{E}_{\mathrm{T}}$ and a radial component $\delta \mathbf{E}_{\mathrm{R}}$. The amplitudes of these two orthogonal oscillations $\left(I_{\mathrm{T}}=\left|\delta \mathbf{E}_{\mathrm{T}}\right|^{2}\right.$ and $\left.I_{\mathrm{R}}=\left|\delta \mathbf{E}_{\mathrm{R}}\right|^{2}\right)$ can be measured separately using a polarizer. The total radiance $I$ and degree of polarization $p$ are defined as follows:

$$
I=I_{\mathrm{T}}+I_{\mathrm{R}}
$$


(a) Two Observers at 0.5 and $1 \mathrm{AU}$
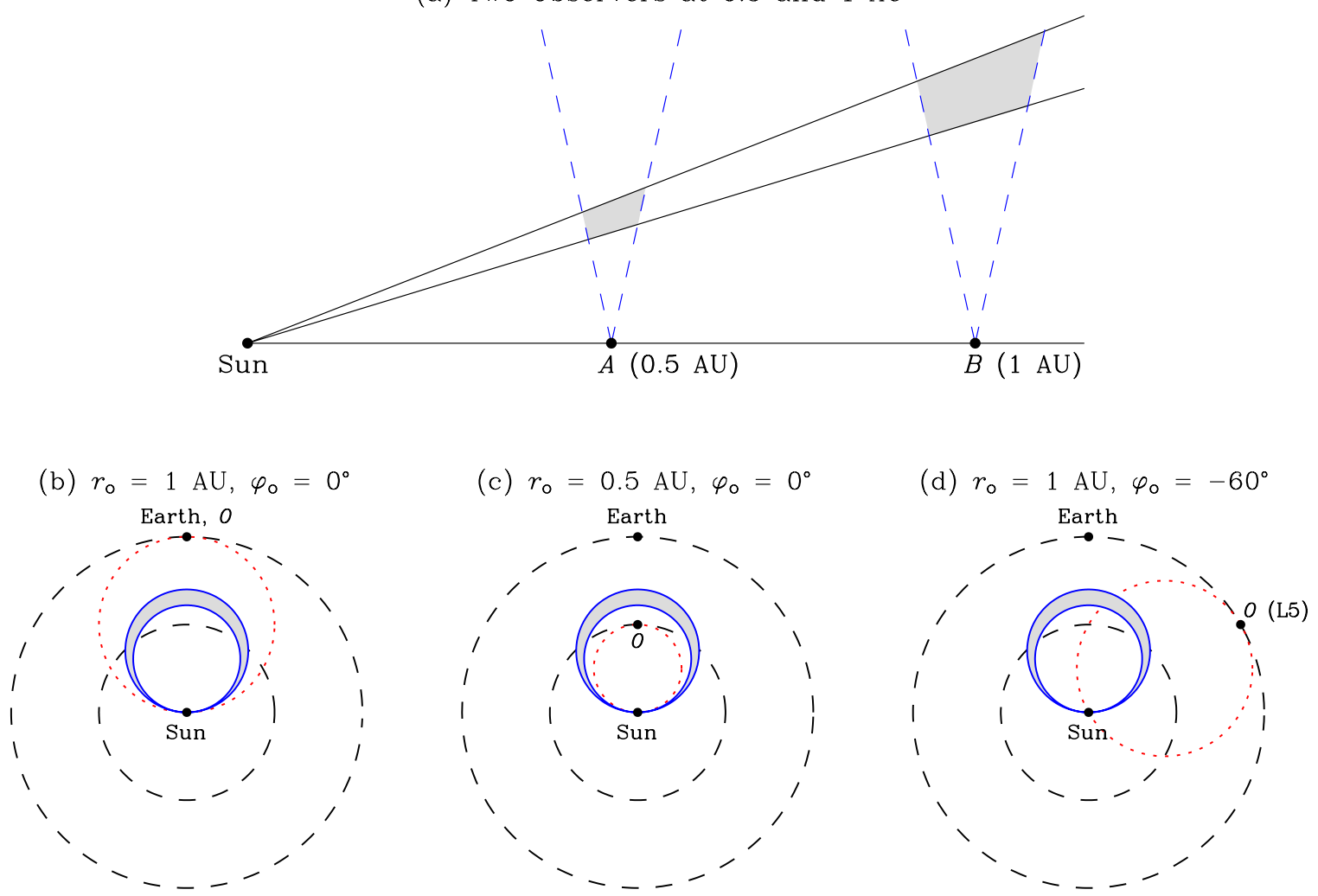

Figure 1. Examples of the Thomson scattering geometry for observers at different radial distances $r_{\mathrm{o}}$ and longitudes $\varphi_{\mathrm{o}}$. In panel (a), a radially propagating solar wind parcel is viewed sequentially, but at the same scattering angle, by observers situated at radial distances of $0.5 \mathrm{AU}$ (point $A$ ) and 1 AU (point $B$ ). Panels (b-d) illustrate the observation of an interplanetary sheath, denoted as a shaded region, by observers with different combinations of $r_{\mathrm{o}}$ and $\varphi_{\mathrm{o}}$. Longitude is defined to be positive (negative) for an observer situated to the west (east) of the Earth.

(A color version of this figure is available in the online journal.)

$$
p=\frac{I_{\mathrm{T}}-I_{\mathrm{R}}}{I} .
$$

Although the incident sunlight is unpolarized $(p=0)$, the scattered WL radiance remains unpolarized only when the scattering angle $\left|\chi-90^{\circ}\right|=90^{\circ}$. The scattered light is elliptically polarized $(0<p<1)$ for $0^{\circ}<\left|\chi-90^{\circ}\right|<90^{\circ}$ and linearly polarized $(p=1)$ for $\chi=90^{\circ}$. Each pixel of a detector records the LOS integral of the local WL radiance:

$$
\left(\begin{array}{c}
I \\
I_{\mathrm{T}} \\
I_{\mathrm{R}}
\end{array}\right)=\int_{0}^{\infty}\left(\begin{array}{c}
i \\
i_{\mathrm{T}} \\
i_{\mathrm{R}}
\end{array}\right) d z=\int_{0}^{\infty} n z^{2}\left(\begin{array}{c}
G \\
G_{\mathrm{T}} \\
G_{\mathrm{R}}
\end{array}\right) d z
$$

Here, $z$ refers to the distance between the detector and the scattering site, as shown in Figure 1(b) of Xiong et al. (2013). The mathematical expressions for $G, G_{\mathrm{R}}$, and $G_{\mathrm{T}}$ are given by Equations (1) and (2) of Xiong et al. (2013). The observed WL radiance is determined jointly by the heliospheric distribution of electrons $n$ and the Thomson scattering geometry factors $\left(z^{2} G\right.$, $z^{2} G_{\mathrm{R}}$, and $z^{2} G_{\mathrm{T}}$ ).

As noted above, the efficiency of Thomson scattering depends strongly on the Thomson scattering angle $\chi$. The perpendicular scattering, $\chi=90^{\circ}$, received by an observer comes from the Thomson sphere. The Thomson sphere, sometimes called the "Thomson surface," is the sphere in which the Sun and observer lie at opposite ends of a diameter (e.g., Vourlidas \& Howard 2006; Howard \& DeForest 2012). The ecliptic cross sections of the Thomson scattering spheres for three observers are shown as dotted circles in Figures 1(b)-(d). The LOS from an observer crosses its Thomson sphere at a so-called $p$ point (Figure 2, Tappin et al. 2004), where both the intensity of incident sunlight and local electron density are greatest, but the efficiency of Thomson scattering is the least. Competition between these three effects results in the spread of local radiance $\left(i, i_{\mathrm{T}}\right.$, and $i_{\mathrm{R}}$ in Equation (3)) to large distances from the Thomson surface, an effect that is greater at larger elongations $\varepsilon$ from the Sun. Howard \& DeForest (2012) described this broad spreading effect using the term "Thomson plateau." Namely, along a single LOS, the radiance per unit electron density is virtually constant over a broad range of scattering angles $\chi$ centered at the $p$ point. The Thomson plateau, in terms of its relevance to heliospheric images, was discussed in detail by Howard \& Tappin (2009), Howard \& DeForest (2012), and Xiong et al. (2013).

A major milestone in stereoscopic WL imaging of interplanetary CMEs was achieved by the STEREO/HI instruments (e.g., Eyles et al. 2009; Davies et al. 2009; Davis et al. 2009; Harrison et al. 2009). This heliospheric imaging capability built on the heritage of the Solar Mass Ejection Imager instrument on the Coriolis spacecraft (Eyles et al. 2003). The STEREO mission is comprised of two spacecraft, with one leading (STEREO A) and the other trailing (STEREO B) the Earth in its orbit. Both spacecraft separate from the Earth by 22.5 per year. The HI instrument on each STEREO spacecraft consists of two cameras, HI-1 and HI-2, whose optical axes lie in the ecliptic. Elongation coverage in the ecliptic is $4^{\circ}-24^{\circ}$ for $\mathrm{HI}-1$ and $18.7-88^{\circ} .7$ for $\mathrm{HI}-2$. The FOV is $20^{\circ} \times 20^{\circ}$ for HI- 1 and $70^{\circ} \times 70^{\circ}$ for $\mathrm{HI}-2$. The cadence of HI-1 is usually 40 minutes and that of $\mathrm{HI}-2$ is $2 \mathrm{hr}$ (Eyles et al. 2009). The current generation of HIs 
do not have WL polarizers. Polarization measurements have, up until now, only been made by coronagraphs (e.g., Poland \& Munro 1976; Crifo et al. 1983; Moran \& Davila 2004; Pizzo \& Biesecker 2004; de Koning et al. 2009; Moran et al. 2010). For instance, Moran \& Davila (2004) used polarization measurements of WL radiance by the $\mathrm{SOHO} / \mathrm{LASCO}$ coronagraph to reconstruct CME orientations near the Sun.

Sky maps, often presented in the Hammer-Aitoff projection, can be used to highlight and track WL transients (e.g., Tappin et al. 2004; Zhang et al. 2013). Time-elongation maps (J-maps) are usually constructed by stacking differenced radiance between observed sky maps along a fixed position angle (sometimes background subtracted images are used instead of difference images). Using such J-maps, transients such as CMEs are manifested as inclined streaks (e.g., Sheeley et al. 2008; Rouillard et al. 2008; Xiong et al. 2011; Harrison et al. 2012; Davies et al. 2012; Xiong et al. 2013). As a propagating transient is viewed at larger elongations, its WL signatures become fainter.

\subsection{Faraday Rotation Formula}

Due to a FR effect, the plane of polarization of linearly polarized radio emission is continuously rotated as the radio wave passes through the heliosphere. For radio waves, the ubiquitous magnetized solar wind flow serves as a magneto-optical birefringence medium. The formulae for FR are expressed below:

$$
\begin{gathered}
\Omega=\Omega_{\mathrm{RM}} \cdot \lambda^{2} \\
\Omega_{\mathrm{RM}}=\int \omega_{\mathrm{RM}} d z \\
\omega_{\mathrm{RM}}=\frac{e^{3}}{8 \pi^{2} \epsilon_{0} m_{\mathrm{e}}^{2} c^{3}} n B_{\|} \\
=\left[2.63 \times 10^{-13} \frac{\mathrm{rad}}{\mathrm{T}}\right] n B_{\|} \\
\delta \omega_{\mathrm{RM}} \propto \delta\left(n B_{\|}\right) \\
\delta \Omega_{\mathrm{RM}} \propto \int \delta \omega_{\mathrm{RM}} d z,
\end{gathered}
$$

where $q, \epsilon, m_{\mathrm{e}}$, and $c$ represent the electron charge, permittivity of free space, mass of an electron, and speed of light constants, respectively. A FR measurement of $\Omega_{\mathrm{RM}}=1 \mathrm{rad} \mathrm{m}^{-2}$ corresponds to $\Omega=0.97$ at $2.3 \mathrm{GHz}$ (wavelength $\lambda=0.13 \mathrm{~m}$ ), $\Omega=57.3$ at $300 \mathrm{MHz}(\lambda=1 \mathrm{~m})$, and $\Omega=1432^{\circ}$ at $60 \mathrm{MHz}$ $(\lambda=5 \mathrm{~m})$. The calibration of ground-based FR observations is difficult since the radio wave passes through the magnetized plasma of the ionosphere, magnetosphere (including the plasmasphere), and solar wind. Oberoi \& Lonsdale (2012) surveyed and compared the FR signatures associated with each of these different regions.

A large portion of the inner heliosphere can be monitored using FR imaging. Prime heliospheric targets measured in FR include interplanetary CMEs and CIRs (Oberoi \& Lonsdale 2012). Because low-frequency radio interferometers such as the MWA, LOFAR, and the VLA feature a wide FOV, high sensitivity, and multi-beam forming capabilities, it is possible to map the magnetic field in the inner heliosphere with a remarkable sensitivity. The high sensitivity of FR measurements enables detections of fluctuations in the heliospheric/interstellar magnetic field and plasma density resulting from MHD turbulence (e.g., Jokipii \& Lerche 1969; Goldshmidt \& Rephaeli 1993; Hollweg et al. 2010). For instance, gradients in FR measurement have been observed across active galactic nucleus jets using the Very Long Baseline Array, which demonstrate that ordered helical magnetic fields are associated with these jets (e.g., Zavala \& Taylor 2002; Gómez et al. 2008; Reichstein \& Gabuzda 2012). The sheath region associated with a fast CME can be similarly probed. FR measurements of the sheath would provide a value for $\Omega_{\mathrm{RM}}$ using Equations (4)-(8). Any measured value of the $\Omega_{\mathrm{RM}}$ would correspond to a statistical average, as the plasma and magnetic fields within such sheath regions are in a highly turbulent state.

\section{WHITE LIGHT AND FARADAY ROTATION SIGNALS RECEIVED AT 0.5 AND 1 AU}

Remote imaging in WL and FR of an Earth-directed sheath from two vantage points, one at $0.5 \mathrm{AU}$ and the other at 1 AU, is considered in Section 3.1. Section 3.2 demonstrates how spatial position and electron number density can be inferred from polarization observations of WL radiance. Section 3.3 presents a means by which the magnetic field can be determined from FR measurements.

\subsection{Comparing Remotely-sensed WL and FR Observations from Different Vantage Points}

Figure 2 shows the modeling results of an Earth-directed sheath propagating from the Sun to $1 \mathrm{AU}$. The traveling sheath is imaged simultaneously by two observers at 0.5 and $1 \mathrm{AU}$. The WL and FR signatures of the sheath are synthesized, using the methods in Section 2. Representative LOSs, which cut through the sheath (LOS1-6), are denoted using arrows in Figure 2. The variations of various physical parameters along LOS1-3 are shown in Figure 3. LOS1, LOS2, LOS3, and LOS5 are approximately tangential to the left flank of the shock; LOS4 and LOS6 are tangential to the nose of the shock. LOS1 and LOS4 are directed toward the observer situated at $0.5 \mathrm{AU}$; all other LOSs are directed toward the observer at 1 AU. The viewing configuration for LOS1 (Figure 2(a)) is equivalent to that for LOS3 (Figure 2(c)), as the elongation of the shock front is the same for LOS1 and LOS3. Thus, the Thomson scattering geometry is identical for these two LOSs, leading to similar LOS profiles in Figure 3. Of course, the observed radiance along LOS1 is much stronger than that along LOS3 (Table 1). Similarly, the observations along LOS4 and LOS6 (Figures 2 (d) and (f)) have identical Thomson scattering geometries. At any given time, the sheath is viewed at greater elongations from a vantage point closer to the Sun. For instance, at an elapsed time of $5.5 \mathrm{hr}$, the foremost elongation $\varepsilon$ of the sheath is $20^{\circ}$ for an observer at $1 \mathrm{AU}(\operatorname{LOS} 1)$ compared with $7^{\circ}$ for an observer at 0.5 AU (LOS2). While the sheath is undetectable in WL along LOS2 (Figure 3(g)), it can be observed in FR (Figure 3(1)). The portion of an LOS that contributes most to the WL radiance broadens and flattens with increasing elongation and shifts gradually toward the observer. At elongations beyond $90^{\circ}$, only back-scattered photons are received; electrons in the vicinity of the observer mainly contribute to remote sensing signatures for elongations beyond $90^{\circ}$. Such observations for elongations of $\varepsilon \geqslant 90^{\circ}$ are less useful for the purposes of space weather prediction. In Figures 2 (d) and (f), the shock front has already reached the observer and can be detected in situ. 

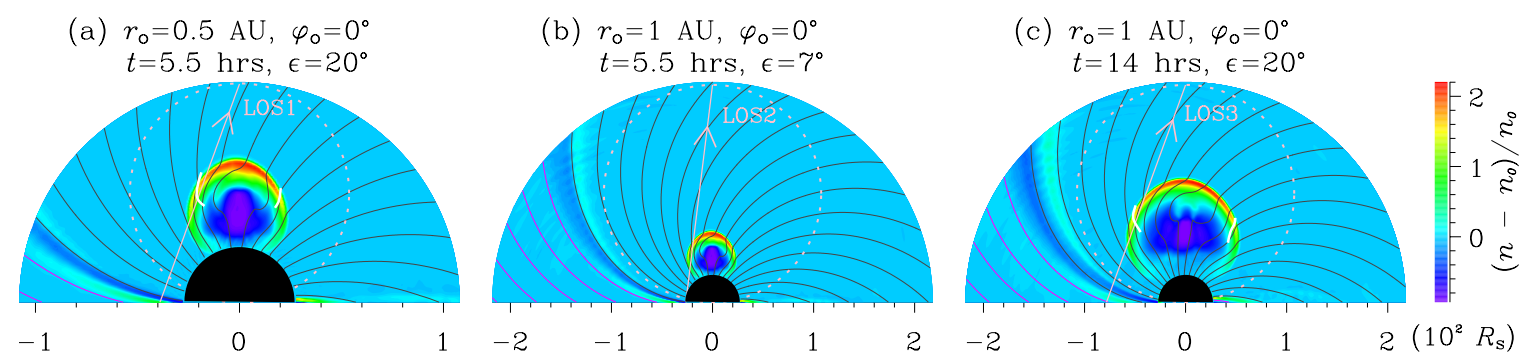

(d) $r_{\mathrm{o}}=0.5 \mathrm{AU}, \varphi_{\mathrm{o}}=0^{\circ}$ $t=11.5 \mathrm{hrs}, \epsilon=70^{\circ}$

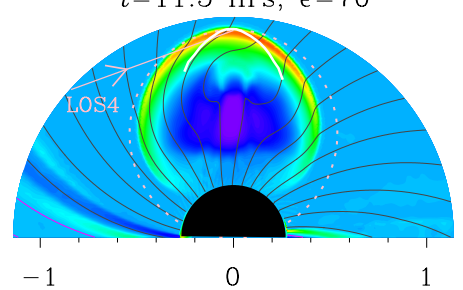

(e) $r_{\mathrm{o}}=1 \mathrm{AU}, \varphi_{\mathrm{o}}=0^{\circ}$ $t=11.5 \mathrm{hrs}, \epsilon=16^{\circ}$

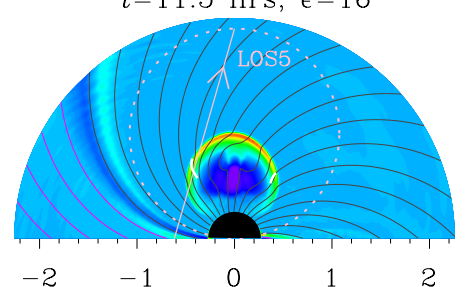

(f) $r_{\mathrm{o}}=1 \mathrm{AU}, \varphi_{\mathrm{o}}=0^{\circ}$

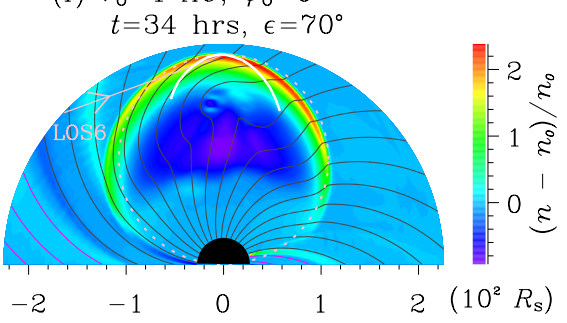

Figure 2. Relative enhancement of the electron number density in the ecliptic $\left(n-n_{0}\right) / n_{0}$, within an Earth-directed interplanetary sheath. The red and black solid lines indicate sunward and anti-sunward interplanetary magnetic field lines, respectively. The sheath is imaged by two observers on the Sun-Earth line $\left(\varphi=0^{\circ}\right)$, at heliocentric distances of $r_{\mathrm{o}}=0.5$ (left column) and $1 \mathrm{AU}$ (middle and right columns). For each observer, the ecliptic cross section of its corresponding WL Thomson scattering sphere is depicted as a dotted circle. Six lines of sight, LOS1-LOS6, are superimposed as straight arrows. All LOSs look westward. At any given time $t$, two observers, both located on the Sun-Earth line, detect the sheath at different elongations $\varepsilon$ (compare panel a with panel b; compare panel d with panel e). Conversely, when viewing along the same $\varepsilon$, the two observers detect the sheath at different $t$ (compare panel a with panel c; compare panel d with panel f). The solid white curves overlaid on each panel indicate the position of the sheath inferred from polarized WL imaging.

(A color version of this figure is available in the online journal.)

Table 1

Maximum Values of the Parameters $i, z^{2} G, z^{2} G_{\mathrm{R}}, z^{2} G_{\mathrm{T}}, n,\left|B_{\|}\right|$, and $\left|\omega_{\mathrm{RM}}\right|$ along LOS1, LOS2, LOS3, and LOS7, Used for Normalization in Figures 3 and 10(e)-(j)

\begin{tabular}{|c|c|c|c|c|c|c|c|c|c|}
\hline LOS & $\begin{array}{c}\text { Time } \\
t \\
(\mathrm{hr})\end{array}$ & $\begin{array}{c}\text { Radii } \\
r_{\mathrm{o}} \\
(\mathrm{AU})\end{array}$ & $\begin{array}{l}\text { Longitude } \\
\qquad \varphi_{\mathrm{o}} \\
\left(^{\circ}\right)\end{array}$ & $\begin{array}{l}\text { Elongation } \\
\qquad \begin{array}{l}\varepsilon \\
\left({ }^{\circ}\right)\end{array}\end{array}$ & $\begin{array}{c}\text { WL Radiance } \\
\quad \begin{array}{c}i \\
\left(\times 10^{-27}\right)\end{array}\end{array}$ & $\begin{array}{l}\text { WL Thomson scattering } \\
\text { Geometry Factors } \\
z^{2} G, z^{2} G_{\mathrm{R}}, z^{2} G_{\mathrm{T}} \\
\left(\times 10^{-29}\right)\end{array}$ & $\begin{array}{l}\text { Electron } \\
\text { Number } \\
\text { Density } n \\
\left(\mathrm{~cm}^{-3}\right)\end{array}$ & $\begin{array}{l}\text { Parallel Magnetic } \\
\text { Field } \\
\left|B_{\|}\right| \\
(\mathrm{nT})\end{array}$ & $\begin{array}{l}\text { FR Measurement } \\
\begin{array}{c}\left|\omega_{\mathrm{RM}}\right| \\
\left(\times 10^{-12} \mathrm{rad} \mathrm{m}^{-3}\right)\end{array}\end{array}$ \\
\hline LOS1 & 5.5 & 0.5 & 0 & 20 & 36.3 & 9.33 & 392 & 207 & 14.4 \\
\hline LOS2 & 5.5 & 1 & 0 & 7 & 344 & 18.2 & 1893 & 223 & 18.2 \\
\hline LOS3 & 14 & 1 & 0 & 20 & 1.93 & 2.31 & 87 & 68.9 & 1.51 \\
\hline LOS7 & 14.5 & 1 & -60 & 34 & 0.52 & 0.86 & 60.3 & 28.5 & 0.42 \\
\hline
\end{tabular}

Note. Each LOS is designated a time $t$, a radius $r_{\mathrm{o}}$, a longitude $\varphi_{\mathrm{o}}$, and an elongation $\varepsilon$ in Columns 2-5; LOSs 1-6 are overlaid on Figure 2 and LOS7 is overlaid on Figure 10.

\subsection{Inferences of Sheath Position from Polarized White Light}

The WL radiance of CMEs is determined by both the electron number density distribution and the Thomson scattering geometry (Equation (3)). The total radiance at a scattering site (i) and its constituent radial $\left(i_{\mathrm{R}}\right)$ and tangential $\left(i_{\mathrm{T}}\right)$ components are associated with Thomson scattering factors $z^{2} G, z^{2} G_{\mathrm{R}}$, and $z^{2} G_{\mathrm{T}}$, respectively. Near the Thomson scattering surface, $z^{2} G_{\mathrm{T}}$ is much larger than $z^{2} G_{\mathrm{R}}$. If a dense parcel of plasma, viewed at large elongations, approaches the Thomson surface, its WL signatures will comprise (1) an increase in $I$, (2) an increase in $I_{\mathrm{T}}$, (3) a decrease in $I_{\mathrm{R}}$, and (4) an increase in the degree of polarization $p$. The variation of $p$ is largest, while that of $I$ is negligible. A plasma parcel's distance from the Thomson sphere has a less significant effect on $I$ at larger elongations. However, the determination of the plasma parcel's location will be more uncertain, if only unpolarized WL observations are available, compared with current operational heliospheric imaging systems. Polarization observations can provide an important clue to the primary scattering site. LOS1 in Figure 2 is used to demonstrate these inferences. The Thomson scattering geometry is independent of the distribution of heliospheric electrons. The degree of polarization $(p)$ and the Thomson scattering factors $\left(z^{2} G, z^{2} G_{\mathrm{R}}\right.$, and $\left.z^{2} G_{\mathrm{T}}\right)$, as presented in Figures 3 (b) and (d), only depend on the modified scattering angle $\chi^{*}=90^{\circ}-\chi$. The profiles of $p, z^{2} G, z^{2} G_{\mathrm{R}}$, and $z^{2} G_{\mathrm{T}}$ are symmetrical around $\chi^{*}=0^{\circ}$. The dependence of $\chi^{*}, z^{2} G$, and the LOS distance $z$ on $p$ can be seen in Figure 4. $p=1$ corresponds to perpendicular scattering (i.e., $\chi^{*}=0^{\circ}$ ). $p \neq 1$ corresponds to two solutions for $\chi^{*}$ : one resulting from forward scattering $\left(\chi^{*}<0^{\circ}\right)$ and the other associated with backward scattering $\left(\chi^{*}>0^{\circ}\right)$. In response to the passage of the shock, the initial radiance components at $t=0, I_{\mathrm{T} 0}$, and $I_{\mathrm{R} 0}$ are enhanced to values denoted by $I_{\mathrm{T}}$ and $I_{\mathrm{R}}$, respectively. The increase in the radiance components defines a so-called modified degree of polarization that we denote by $p^{*}$, given by

$$
p^{*}=\frac{I_{\mathrm{T}}-I_{\mathrm{T} 0}-I_{\mathrm{R}}+I_{\mathrm{R} 0}}{I-I_{0}} .
$$

$p$ is 0.62 along LOS1 at $t=0 \mathrm{hr}$, effectively defining the degree of polarization associated with the background solar 
(A) LOS1
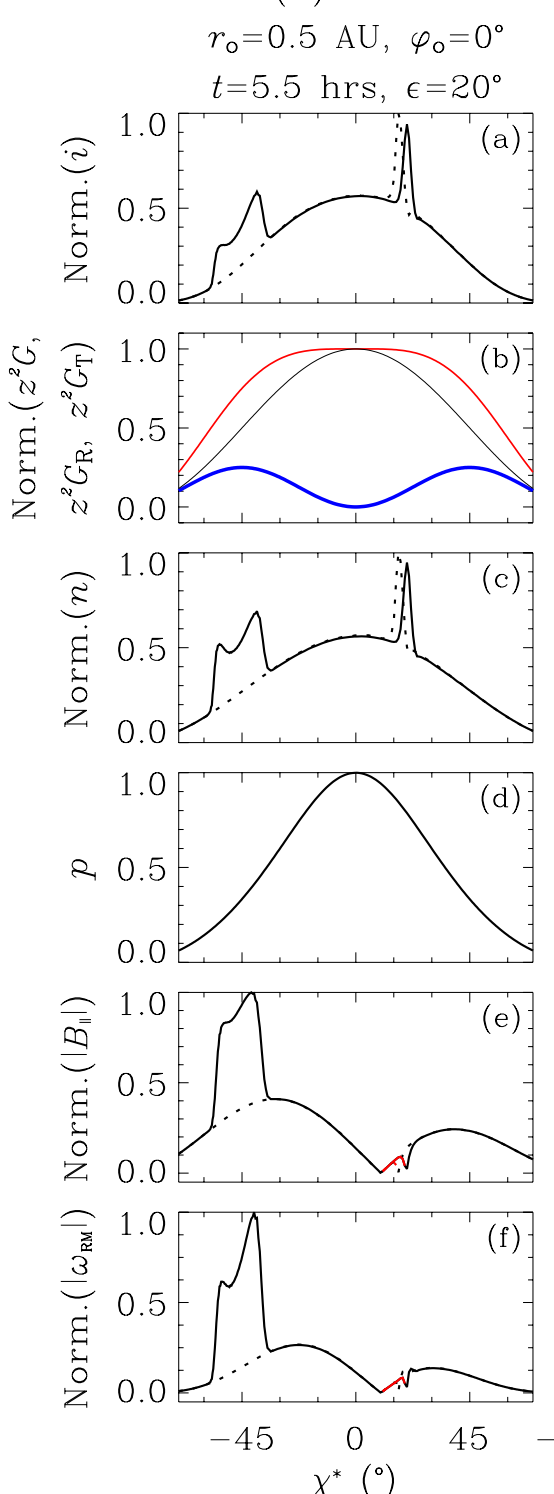

(B) LOS2
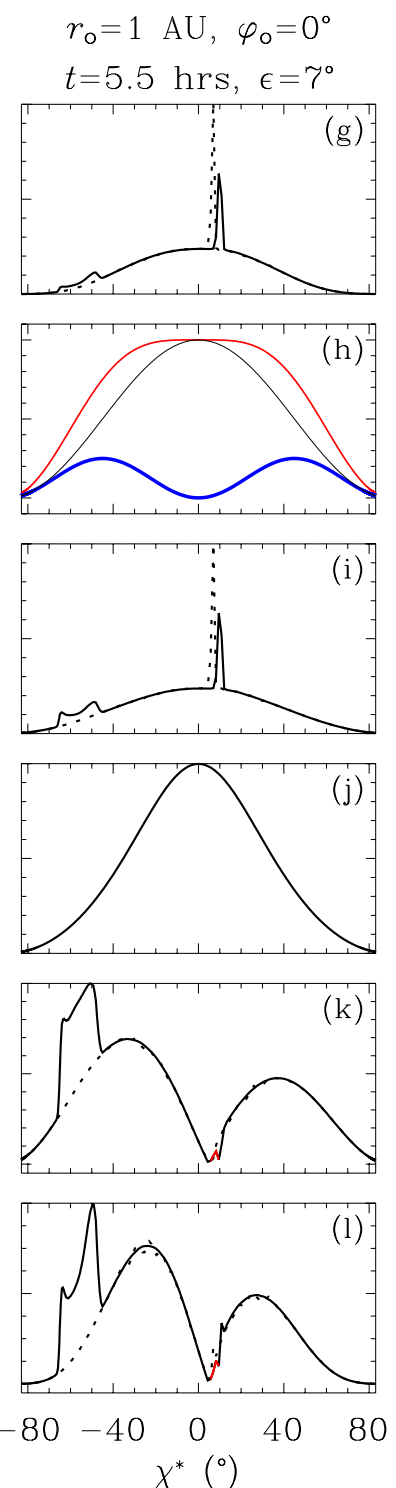

(C) LOS3
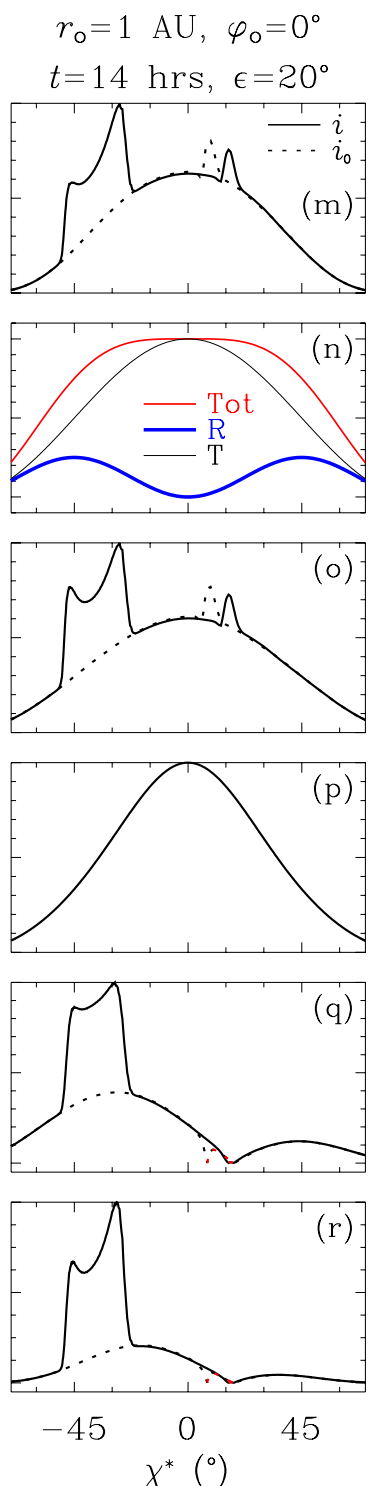

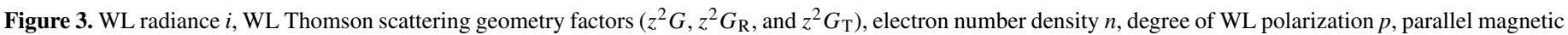

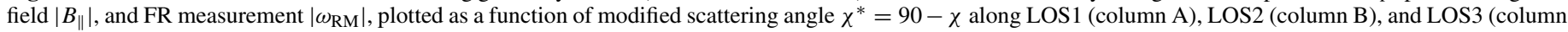

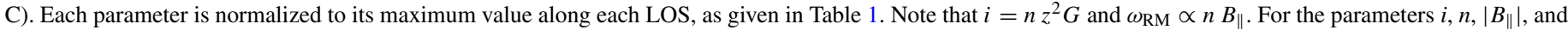

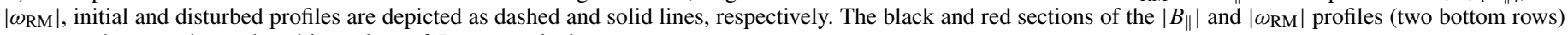
correspond to negative and positive values of $B_{\|}$, respectively.

(A color version of this figure is available in the online journal.)

wind. During the sheath passage, at $t=5.5 \mathrm{hr}, p$ is 0.58 along this LOS. The modified degree of polarization $p^{*}$, derived using Equation (9), is therefore 0.29 at $t=5.5 \mathrm{hr}$. The radiance enhancement is due to the presence of the sheath in the LOS. The sheath, which trails the shock front, occupies a relatively small volume of interplanetary space. The sheath occupies the portion of LOS1 bounded by $-55^{\circ}<\chi^{*}<-35^{\circ}$ (Figures 3 (a) and (c)). Within this region, $p$ smoothly varies from 0.15 to 0.5 (Figure 3(d)). The average value of $p^{*}$ within the sheath is 0.29 . In an inverse approach, $p^{*}$ can be used to estimate the scattering angle $\chi^{*}$ within the sheath, as seen in Figure $4(\mathrm{a}) \cdot p^{*}=0.29$ corresponds to $\chi^{*}= \pm 46^{\circ}$ and $\overline{z^{2} G}=6.5 \times 10^{-29}$, where $\overline{z^{2} G}$ is the average value of $z^{2} G$ in the sheath. The solution of $\chi^{*}=46^{\circ}$ can be immediately excluded, as an Earth-directed $\mathrm{CME}$ can generally be identified (indeed much earlier) as being front-sided based on extreme-ultraviolet images of the full solar disk (e.g., Thompson et al. 1998; Plunkett et al. 1998). The other solution, $\chi^{*}=-46^{\circ}$, is physical and yields a value of $60 R_{\mathrm{S}}$ for the distance, $z$, of the main scattering site (corresponding to the sheath) from the detector. How best to judge which solutions for $\chi^{*}$ are physical is explained in detail in Section 4. Once the Thomson scattering factor $z^{2} G$ of the sheath has been inferred, its column-integrated electron number density can be estimated based on the following equation:

$$
\delta N_{\text {sheath }}=\int \delta n d z \simeq \frac{\int \delta n z^{2} G d z}{\overline{z^{2} G}} .
$$

It is clear that WL polarization measurements can prove extremely valuable in the study of interplanetary CMEs and shocks. 
LOS 1

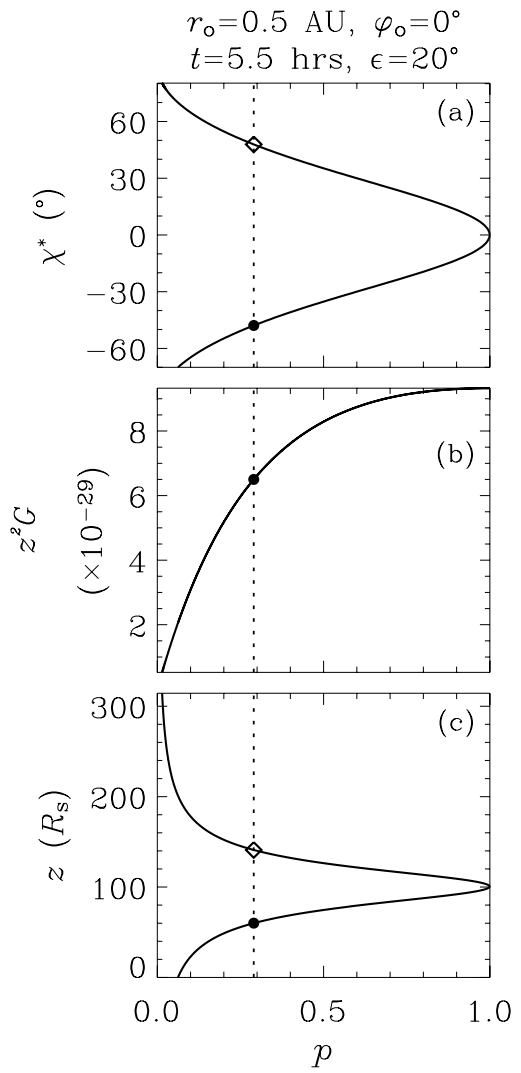

Figure 4. Dependence of the scattering angle $\chi^{*}$, the Thomson scattering geometry factor $z^{2} G$, and the LOS depth $z$ on the degree of polarization $p$ for LOS1 in Figure 2(a). In response to the sheath passage, the initial radiance components, $I_{\mathrm{T} 0}$ and $I_{\mathrm{R} 0}$, are enhanced to values of $I_{\mathrm{T}}$ and $I_{\mathrm{R}}$, respectively. The enhancement in these radiance components determines the modified degree of polarization $p^{*}$, according to the expression $p^{*}=\left(I_{\mathrm{T}}-I_{\mathrm{T} 0}-I_{\mathrm{R}}+I_{\mathrm{R} 0}\right) /\left(I-I_{0}\right)$. The vertical dashed line, denoting $p^{*}$, crosses the $\chi^{*}$ and $z$ profiles twice.

\subsection{The Magnetic Field Inferred from Faraday Rotation}

As discussed in Section 3.2, the column-integrated electron number density along any LOS can be inferred from WL observations. Thus, if a radio beam lies within the FOV of a WL imager such that both instruments remotely probe the same plasma volume, the WL density measurements can be used to extract the magnetic field strength from the received FR signal. We demonstrate this technique for LOS1 in Figure 5. After subtracting the background solar wind contribution, the enhancements in FR measurement and WL radiance due to the presence of the sheath of the simulated Earth-directed shock are given by $\delta \Omega_{\mathrm{RM}}$ and $\delta I$, respectively. The ratio of $\delta \Omega_{\mathrm{RM}}$ and $\delta I$ can be expressed as

$$
\begin{aligned}
\frac{\delta \Omega_{\mathrm{RM}}}{\delta I} & =\frac{\int \delta \omega_{\mathrm{RM}} d z}{\int \delta i d z}=\frac{\int \delta\left(n B_{\|}\right) d z}{\int z^{2} G \delta n d z} \\
& \approx \frac{\int \delta\left(n B_{\|}\right) d z}{\overline{z^{2} G} \int \delta n d z} \approx \frac{1}{\overline{z^{2} G}} \frac{\delta\left(n B_{\|}\right)}{\delta n} .
\end{aligned}
$$

As discussed in Section 3.2, $\overline{z^{2} G}$ corresponds to the average value of $z^{2} G$ in the sheath. The derivable parameter $\delta\left(n B_{\|}\right) / \delta n$, which we call $B_{\|}^{*}$, can be expressed in the form

$$
B_{\|}^{*} \equiv \frac{\delta\left(n B_{\|}\right)}{\delta n}=\delta B_{\|}+B_{\| 0}+n_{0} \frac{\delta B_{\|}}{\delta n}=B_{\|}+n_{0} \frac{\delta B_{\|}}{\delta n}>B_{\|},
$$

LOS1

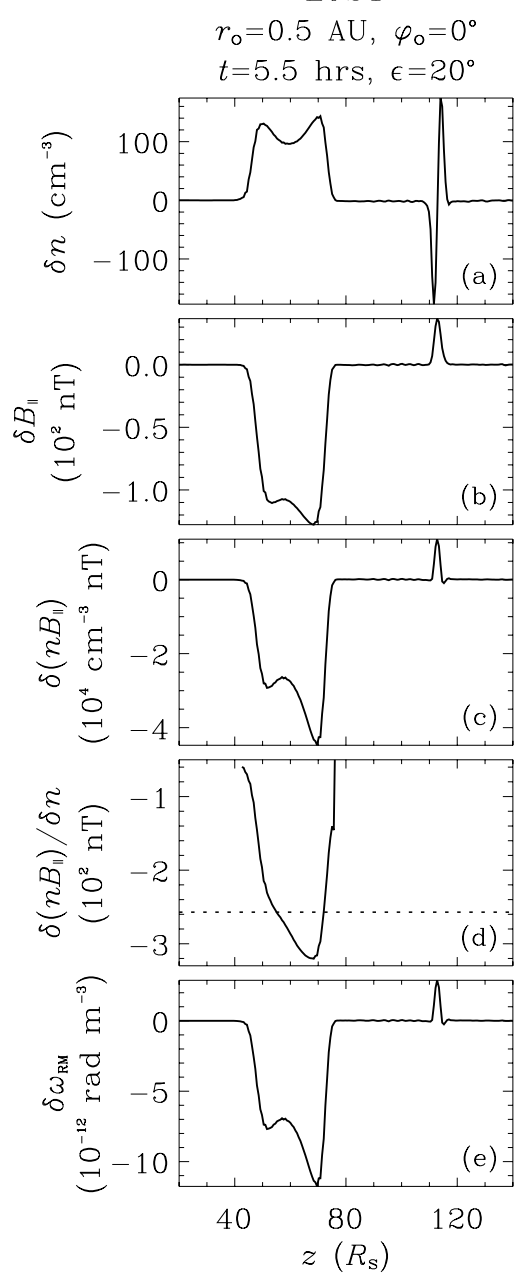

Figure 5. Deviation of various parameters from their initial values, plotted as a function of $z$ along LOS1. Note that $\left(\delta\left(n B_{\|}\right) / \delta n\right)=\delta B_{\|}+$ $B_{\| 0}+n_{0}\left(\delta B_{\|} / \delta n\right)=B_{\|}+n_{0}\left(\delta B_{\|} / \delta n\right)$. The parallel magnetic field component $B_{\|}^{*}$ (plotted as a horizontal dashed line in panel d) is calculated using the expression $\left(\delta \Omega_{\mathrm{RM}} / \delta I\right)=\left(\left(\int \delta \omega_{\mathrm{RM}} d z\right) /\left(\int \delta i d z\right)\right)=$ $\left(\left(\int \delta\left(n B_{\|}\right) d z\right) /\left(\int z^{2} G \delta n d z\right)\right) \approx\left(1 / \overline{z^{2} G}\right)\left(\delta\left(n B_{\|}\right) / \delta n\right)=\left(1 / \overline{z^{2} G}\right) B_{\|}^{*}$.

where $B_{\| 0}$ and $n_{0}$ denote the initial background values of $B_{\|}$ and $n$, respectively. The inferred value of $B_{\|}^{*}$ serves as an upper limit on $B_{\|}$.

\section{RADIANCE PATTERNS IN J-MAPS OF WHITE LIGHT AND FARADAY ROTATION}

Shock propagation through the inner heliosphere can be identified through the inclined trace with which it is associated in time-elongation maps (J-maps). J-maps of WL radiance $\left(I / I^{*}, I\right)$, the degree of polarization $\left(p, p^{*}\right)$, and FR measurements $\left|\delta \Omega_{\mathrm{RM}}\right|$, as viewed by observers at 0.5 and $1 \mathrm{AU}$, are presented in Figure 6 and compared in Figure 7. The normalization factor $I^{*}$ in Figure 6 corresponds to an electron number density distribution that varies according to $n \propto r^{-2}$. A radiance threshold of $I / I^{*} \geqslant 3.68 \times 10^{-15}$ is used to demarcate the sheath region in time-elongation $(t-\varepsilon)$ parameter space. The modified polarization $p^{*}$ is only calculated, using Equation (9), inside the sheath (Figures $6(\mathrm{~g})-(\mathrm{h})$ ). The absolute values of $I$ and $\left|\delta \Omega_{\mathrm{RM}}\right|$ within the sheath region are much larger for the observer at $0.5 \mathrm{AU}$, whereas the sheath values of $I / I^{*}, p$, and $p^{*}$ are comparable when viewed from either vantage point. 

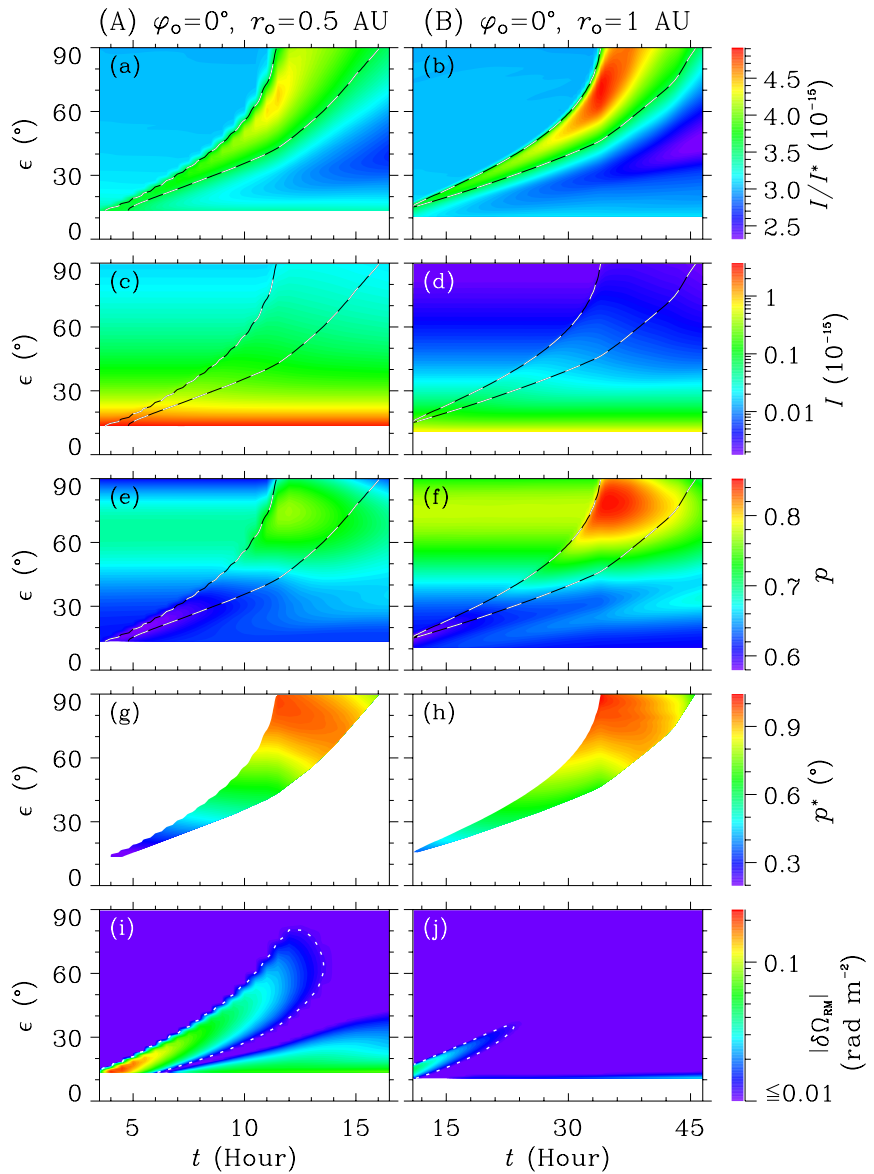

Figure 6. Time-elongation maps of WL radiance (panels a and b: $I / I^{*}$; panels c and $\mathrm{d}: I$ ), degree of WL polarization (panels e and $\mathrm{f}$ : $p$; panels $\mathrm{g}$ and $\mathrm{h}: p^{*}$ ), and FR measurements (panels $i$ and $\mathrm{j}:\left|\delta \Omega_{\mathrm{RM}}\right|$ ), as viewed by observers at a longitude $\varphi_{\mathrm{o}}=0^{\circ}$ and at radii $r_{\mathrm{o}}=0.5 \mathrm{AU}$ (left column) and $1 \mathrm{AU}$ (right column). $I^{*}$ is the normalization factor for $I$ and corresponds to the radial variation of the electron number density $n \propto r^{-2}$. The dotted lines in panels a-f correspond to $I / I^{*}=3.68 \times 10^{-15} \cdot p$ and $p^{*}$ are determined from the radiance and the enhancement in the radiance, respectively, as given by $p=\left(I_{\mathrm{T}}-I_{\mathrm{R}}\right) / I$ and $p^{*}=\left(I_{\mathrm{T}}-I_{\mathrm{T} 0}-I_{\mathrm{R}}+I_{\mathrm{R} 0}\right) /\left(I-I_{0}\right)$. Panels $\mathrm{g}$ and $\mathrm{h}$ only show $p^{*}$ within the sheath region.

(A color version of this figure is available in the online journal.)

Over the elongation range $15^{\circ} \leqslant \varepsilon \leqslant 180^{\circ}$, the radiance ratio $\left(\right.$ Max. $\left.\left(I_{0.5 \mathrm{AU}}\right)\right) /\left(\right.$ Max. $\left.\left(I_{1 \mathrm{AU}}\right)\right)$ is limited to values between 8 and 11 (Figure 7(c)). This fact demonstrates that interplanetary CMEs and shocks are better viewed from a location closer to the Sun.

The position, mass, and magnetic field of the sheath can be inferred from those directly measurable parameters presented in Figure 6, using the analytical methods presented in Sections 2.1 and 3.3. As shown in Figure 4 and explained in Section 3.2, the Thomson scattering factors are symmetrical around $\chi^{*}=0^{\circ}$. As a result, a single value of $p^{*}$ corresponds to two symmetrical solutions for a scattering angle $\chi^{*}$. The results shown in Figure 8 are derived from those in Figure 6 (for an observer at 0.5 $\mathrm{AU}$ ) under the assumption of forward scattering, while those in Figure 9 assume backward scattering. For the forward-scattering situation, presented in Figure 8(b), the inferred longitude of the sheath $\varphi_{\text {sheath }}$ is $22^{\circ}$ at an elapsed time of $5 \mathrm{hr}$ and $9^{\circ}$ at 11 hr. For the backward-scattering case, shown in Figure 9(b), the sheath is at $\varphi_{\text {sheath }}=140^{\circ}$ and $60^{\circ}$ at these times. So, an observer at $0.5 \mathrm{AU}$ infers a longitude change of $\Delta \varphi_{\text {sheath }}=$ $13^{\circ}$ (Figure 8(b)) and $80^{\circ}$ (Figure 9(b)) for the forward- and
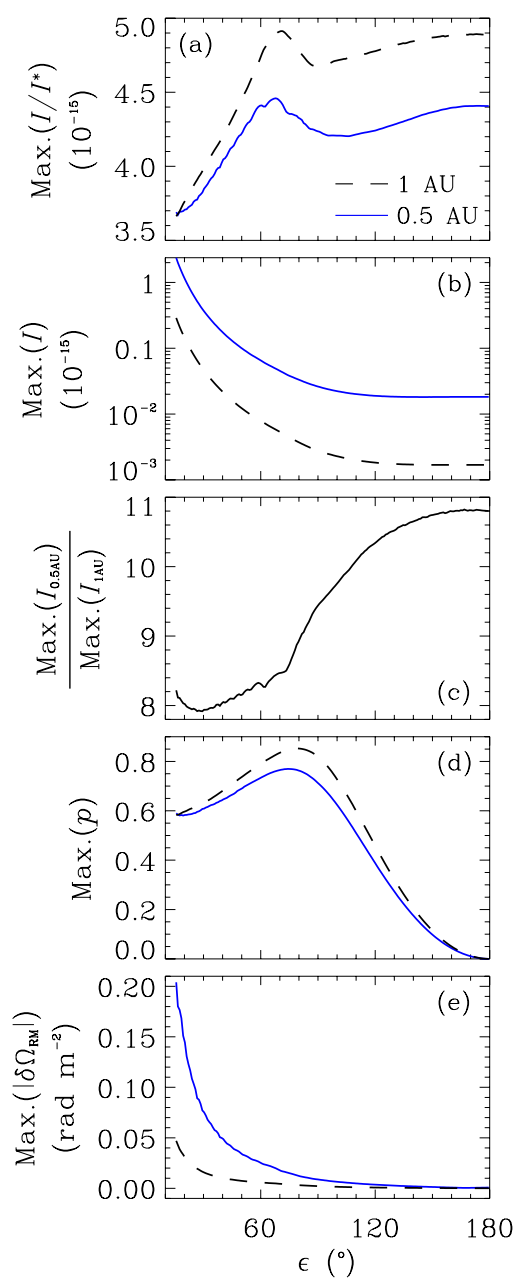

Figure 7. Comparison of the WL radiance $I$ (panels a, b, and c), the degree of WL polarization $p$ (panel d), and the FR measurements $\delta \Omega_{\mathrm{RM}}$ (panel e) over the elongation range of $15^{\circ}$ to $180^{\circ}$, as viewed by observers at $0.5 \mathrm{AU}$ (solid line) and $1 \mathrm{AU}$ (dashed line). The attribution "Max" refers to the strongest signal during the sheath passage.

(A color version of this figure is available in the online journal.)

backward-scattering cases, respectively. The dramatic change in sheath longitude for the backward-scattering case might indicate that the shock is significantly deflected during its interplanetary propagation. However, such an abnormal degree of lateral deflection of $\Delta \varphi_{\text {sheath }}=80^{\circ}$ would be highly unphysical and may imply a "ghost trajectory" (Figure 6, DeForest et al. 2013). The east-west symmetry of the radiance pattern suggests that the shock is actually front-sided and Earth-directed, rendering the assumption of backward-scattering invalid (Figure 9). If we assume that the radiance pattern shown in Figure 6 is attributable to forward scattering, the inferred position of the sheath is shown as the solid white curve in Figure 2. This result agrees very well with the actual position of the sheath. At any given time, only a certain portion of the sheath will be visible from a fixed observing location (Xiong et al. 2013). For example, at an elapsed time of $5.5 \mathrm{hr}$, it is the flank of the sheath (Figure 2(a)) that corresponds to the leading edge of the radiance pattern in Figure 6(a), while $6 \mathrm{hr}$ later it is the nose (Figure 2(d)). So, in fact, the longitudinal change of $\Delta \varphi_{\text {sheath }}=13^{\circ}$ inferred from Figure 8(b) is actually an artifact of the viewing geometry and does not represent an actual deflection of the shock front. Along with the inferred position of the sheath, the column-integrated electron number density, $\delta N_{\text {sheath }}$, and 

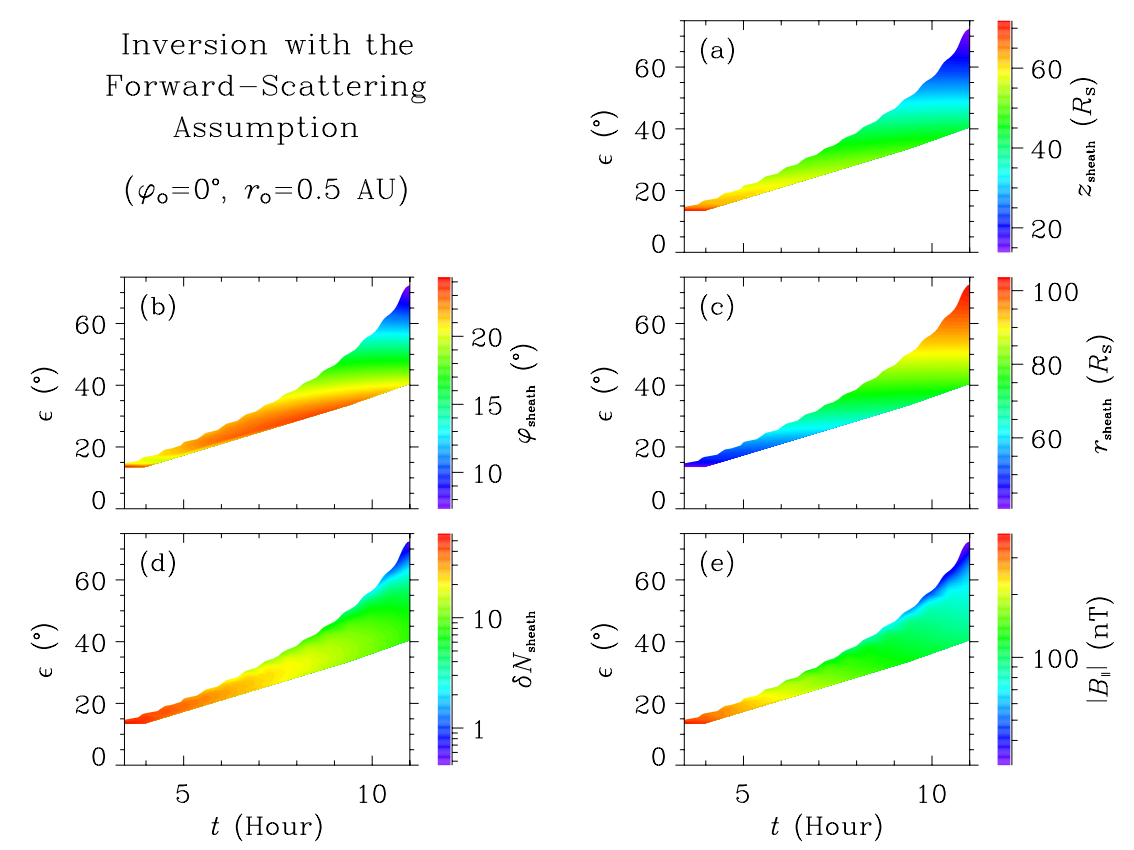

Figure 8. Panels a-e present the location of the scattering site $\left(z_{\text {sheath }}, \varphi_{\text {sheath }}\right.$, and $\left.r_{\text {sheath }}\right)$, the column-integrated electron number density $\delta N_{\text {sheath }}$, and the parallel magnetic field $B_{\|}$, plotted as a function of time and elongation, derived by assuming forward scattering $\left(\chi^{*}<0^{\circ}\right)$.

(A color version of this figure is available in the online journal.)

Inversion with the Backward-Scattering Assumption $\left(\varphi_{\mathrm{o}}=0^{\circ}, r_{\mathrm{o}}=0.5 \mathrm{AU}\right)$
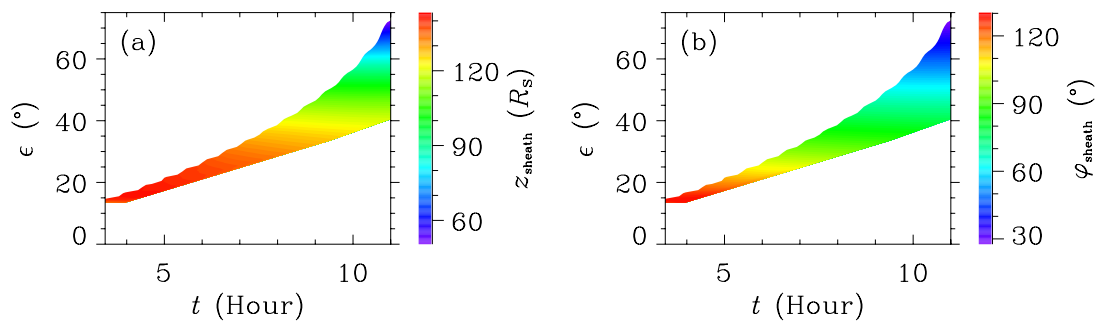

Figure 9. The location of the scattering site $\left(z_{\text {sheath }}, \varphi_{\text {sheath }}\right)$, plotted as a function of time and elongation, derived by assuming backward scattering $\left(\chi^{*}>0^{\circ}\right)$. (A color version of this figure is available in the online journal.)

the parallel magnetic field component, $B_{\|}$, are also presented in Figure 8. The derived value of $\left|B_{\|}\right|$provides an upper limit on the actual magnetic field, as explained in Section 3.3. By making coordinated observations in WL and FR, CMEs can not only be continuously tracked, but quantitatively diagnosed as they propagate through interplanetary space.

\section{INTERPLANETARY IMAGING FROM DIFFERENT OBSERVATION SITES}

An interplanetary CME looks different when viewed from different vantage points, but can be readily imaged from a wide range of longitudes. The observed WL radiance pattern depends not only on the longitude $\varphi_{\mathrm{o}}$ of the observer, as discussed by Xiong et al. (2013), but also on heliocentric distance $r_{\mathrm{o}}$. In Section 3.1, we compared observations made from radial distances of 0.5 and 1 AU. In Section 5.1, we consider two particular observation sites that are often considered favorable in terms of WL imaging, the L4 and L5 Lagrangian points. In Section 5.2, we quantify more fully the dependence of WL imaging on $r_{\mathrm{o}}$.

\subsection{Observing an Earth-directed Shock from the L4 and L5 Lagrangian Points}

The L4 and L5 Lagrangian points of the Sun-Earth system are often considered advantageous for observing Earth-directed CMEs. There are five Lagrangian points, all in the ecliptic, i.e., L1-L5. A spacecraft at L1, L2, or L3 is metastable in terms of its orbital configuration, and hence must frequently use propulsion to remain in the same orbit. In contrast, a spacecraft at L4 or L5 is resistant to gravitational perturbations and is believed to be more stable. The L4 and L5 points lie $60^{\circ}$ ahead of and behind the Earth in its orbit, respectively. STEREO A reached the L4 point in 2009 September and STEREO B reached L5 in 2009 October. The twin STEREO spacecraft were pathfinders for future L4/L5 missions (Akioka et al. 2005; Biesecker et al. 2008; Gopalswamy et al. 2011). A spacecraft at either L4 or L5 can perform routine side-on imaging of Earthdirected CMEs, and hence is of great merit for space weather monitoring.

Figure 10(a) illustrates the imaging, be it in WL or FR, of an Earth-directed sheath from the L5 point. LOS7 intersects the nose of the shock at an elapsed time of $14.5 \mathrm{hr}$, when the shock 

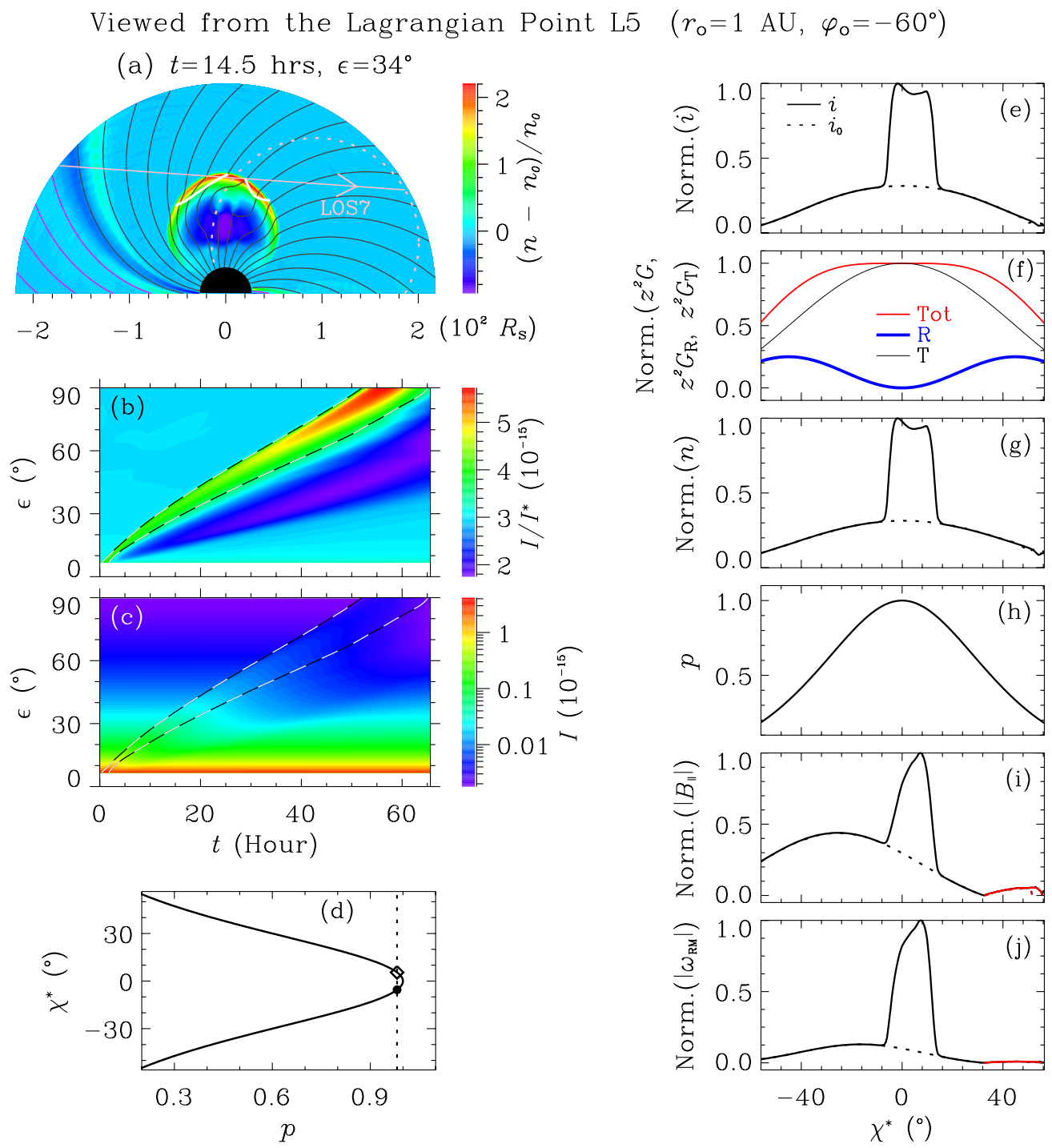

Figure 10. Simulated results corresponding to the observation of an Earth-directed shock from the L5 vantage point, i.e., along LOS7 $\left(r_{\mathrm{o}}=1 \mathrm{AU}, \varphi_{\mathrm{o}}=-60^{\circ}\right)$ : (panel a) relative enhancement of electron number density $\left(n-n_{0}\right) / n_{0}$ in the ecliptic, (panels b and c) synthesized WL time-elongation maps of $I / I^{*}$ and $I$, (panel d) modified scattering angle $\chi^{*}$ as a function of the degree of polarization $p$, and (panels $\mathrm{e}-\mathrm{j}$ ) a number of synthesized WL and FR parameters, plotted as a function of $\chi^{*}$ along LOS7.

(A color version of this figure is available in the online journal.)

nose lies on the Thomson scattering sphere. The variation along LOS7 of a number of salient physical parameters is shown in Figures 10 (e)-(j). The interplanetary magnetic field lines are compressed and rotated within the sheath. This rotation results in the closer alignment of the field lines with LOS7, such that the magnetic field component along the LOS, $\left|B_{\|}\right|$, is greatly enhanced (Figure 10(i)). The enhancements of both $\left|B_{\|}\right|$and electron number density $n$ within the sheath are responsible for the resulting increases in WL radiance $I$ and FR measurement $\left|\Omega_{\mathrm{RM}}\right|$. The degree of WL polarization $p$ that is at an elongation of $34^{\circ}$, as viewed along LOS7, is 0.67 for the background solar wind and increases to 0.75 during the shock passage at $14.5 \mathrm{hr}$. This value corresponds to a value of the modified WL polarization $p^{*}$ of 0.98 , based on Equation (9). As was done for LOS1 in Section 3, we evaluate the WL radiance along LOS7 (Figure 10(a)), from which we infer the shock position (Figure 10(d)). Again, a single value of $p^{*}$ corresponds to two symmetrical solutions for a scattering angle $\chi^{*}$, i.e., $p^{*}=0.29$ and $\chi^{*}= \pm 46^{\circ}$ for LOS1 and $p^{*}=0.98$ and $\chi^{*}= \pm 5^{\circ}$ for LOS7. For LOS1, only one solution, for $\chi^{*}=-46^{\circ}$, was deemed physical; for LOS7, both solutions for $\chi^{*}$ are potentially physical. The scattering sites corresponding to $\chi^{*}= \pm 5^{\circ}$ are very close to one another and both agree well with the actual position of the sheath (Figure 10(a)). The section of LOS7 bounded by $-5^{\circ} \leqslant \chi^{*} \leqslant 5^{\circ}$ lies within the sheath. Both forward scattering $\left(-5^{\circ} \leqslant \chi^{*}<0^{\circ}\right)$ and backward scattering $\left(0^{\circ}<\chi^{*} \leqslant 5^{\circ}\right)$ will contribute to the radiance $I$ observed along this LOS. The propagating sheath can be tracked continuously and easily in WL from the L5 vantage point, since it leaves an obvious signature in the J-map of synthesized radiance (Figures 10 (b) and (c)). This fact confirms previous assertions that the L4 and L5 points are very favorable in terms of space weather monitoring.

\subsection{Dependence of White-light Radiance on Heliocentric Distance}

The background intensity at a fixed elongation in a WL sky map is greater for an observer closer to the Sun. For an HI at any distance from the Sun, Jackson et al. (2010) proposed the 

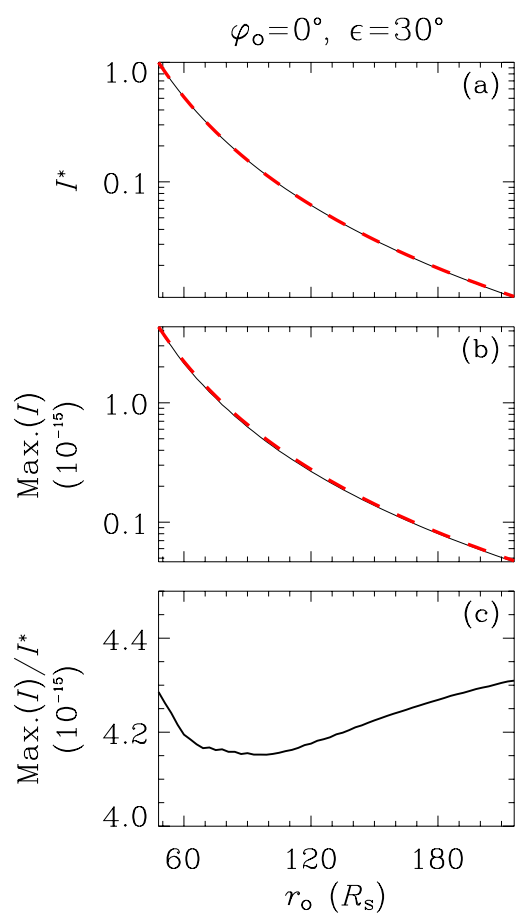

Figure 11. Simulated WL radiance, as a function of $r_{\mathrm{o}}$, viewed along an elongation of $30^{\circ}$ by an observer on the Sun-Earth line $\left(\varphi_{\mathrm{o}}=0^{\circ}, 50 R_{\mathrm{S}} \leqslant r \leqslant\right.$ $\left.215 R_{\mathrm{S}}\right)$. Again, "Max" refers to the strongest signal associated with the sheath passage. The dashed red lines in the two upper panels show, for comparison, an $r^{-3}$ variation. Both $I^{*}$ and Max. $(I)$ are well described by such a variation.

(A color version of this figure is available in the online journal.)

following Thomson scattering principles: (1) the WL radiance $I$ at a given solar elongation falls off with the heliocentric distance $r$ according to $r^{-3}$ and (2) such a dependence of $I \propto r^{-3}$ is valid for almost any viewing elongation and for any radial distance from $0.1 \mathrm{AU}$ out to $1 \mathrm{AU}$ and beyond. The WL radiance $I$ depends on the heliospheric distribution of electron number density $n$. In interplanetary space, the background solar wind speed is nearly constant and the background electron number density $n_{0}$ varies approximately with $r^{-2}$. However, the equilibrium defined by $n_{0} \propto r^{-2}$ is disturbed by the presence of interplanetary transients such as CMEs and CIRs. A traveling shock can sweep up, and hence compress significantly, the background solar wind plasma. Figures 2 and 10(a) show a density enhancement of $\left(n-n_{0} / n_{0}\right) \approx 2.2$ within the sheath. The associated compression ratio $\left(n / n_{0}\right) \approx 3.2$ indicates that the shock is very strong. However, when viewed along elongations less than $60^{\circ}$, the strongest signatures of shock passage (characterized by Max. $(I)$ ) vary very closely with $r^{-3}$ (Figures 11 (b) and (c)). The relationship of Max.(I) $\propto r^{-3}$ is slightly violated at large elongations $\left(\varepsilon>60^{\circ}\right)$. Figure $7(\mathrm{c})$ reveals that the ratio between Max. $\left(I_{0.5 \mathrm{AU}}\right)$ and Max. $\left(I_{1 \mathrm{AU}}\right)$ is close to 8 for $\varepsilon \leqslant 60^{\circ}$, increasing thereafter to 10.8 at $\varepsilon=180^{\circ}$. The premise that the WL radiance decreases with the third power of Sun-observer distance generally holds true for both the background solar wind and propagating CMEs.

\section{DISCUSSION}

The detectability in WL of a particular electron density feature is determined by its signal above the noise background. In the STEREO/HI-1 FOV, the dominant WL signal is zodiacal light due to scattering of sunlight from the F-corona, which is centered around the ecliptic. In STEREO/HI-2 images, the noise floor is primarily determined by photon noise and the background star field (DeForest et al. 2011). Away from the ecliptic, the background WL noise has a sharp radial gradient in coronal images and is nearly constant in heliospheric images. The signal-to-noise ratio for heliospheric electron density features is discussed by Howard et al. (2013). We will address the detection of CMEs in the presence of background noise in future forward modeling work.

If both a transient CME and background (heliospheric current sheet (HCS)-heliospheric plasma sheet (HPS)) plasma structures are present along the same LOS, both will contribute to the total LOS-integrated radiance. In this case, the interpretation of the data would clearly be more problematic. Moreover, if the LOS was to penetrate a HCS, the magnetic field vector would, at that point, rotate through $180^{\circ}$. Due to the mutual cancellation of $B_{\|}$across the HCS, there may be no net FR signature according to Equations (4)-(6). Hence, even such a significant interplanetary structure may be associated with only a weak FR measurement. Conversely, the relatively dense plasma within a HPS can significantly contribute to the WL radiance. Thus, the potential effects of the presence of HCS-HPS structures need to be borne in mind when obtaining remote images of CMEs. In the current work, however, we find that such effects are negligible. In our numerical simulation, there are two HCS-HPS structures, which are initially rooted at longitudes of $\varphi= \pm 90^{\circ}$ at the inner boundary of our numerical simulation. The simulated shock emerges at a longitude of $\varphi=0^{\circ}$. The large longitudinal difference between the HCS-HPS and the shock means that the remote sensing signatures are principally contributed by the sheath. Thus, in our forward modeling work, the signal enhancements of synthesized imaging in WL and FR are unambiguously the result of the propagating sheath.

In general, the more complex the interplanetary dynamics are, the more complex the resulting remote sensing observations will be. For instance, a CME can interact with other CMEs and/or background solar wind structures such as CIRs, HCSs, and HPSs; mutual interactions between CMEs are, however, generally more perturbing than interactions between CMEs and such background structures. Interactions can result in the background solar wind structures becoming warped or distorted (e.g., Odstrčil et al. 1996; Hu \& Jia 2001) and CMEs being accelerated/decelerated (e.g., Lugaz et al. 2005; Xiong et al. 2007; Shen et al. 2012b), deflected (e.g., Xiong et al. 2006b, 2009; Lugaz et al. 2012), distorted (e.g., Xiong et al. 2006b, 2009), or entrained (e.g., Rouillard et al. 2009b). In particular, during such interactions, the behavior of a sheath can become much more complex: the shock aphelion can be deflected, spatial asymmetries can develop along the shock front, and the shock front can potentially merge completely with other shock fronts. At an interaction site, both the plasma density and magnetic field would be compressed, leading to enhanced signatures in both WL and FR observations. For example, the interaction between two CMEs was manifested as a very bright arc in WL images (e.g., Harrison et al. 2012; Liu et al. 2012; Temmer et al. 2012). Different types of interactions would likely result in different WL radiance signatures; in fact, through a single interaction, the corresponding radiance pattern could evolve. The interpretation of such complex WL radiance patterns would be prone to large uncertainties, but can be rigorously constrained if interplanetary imaging is performed from multiple vantage points and complemented by numerical modeling. For stereoscopic WL imaging, ray paths from one observer intersect those from the other observer. Thus, the 
$3 \mathrm{D}$ distribution of electrons in the inner heliosphere can be reconstructed using a time-dependent tomography algorithm (Jackson et al. 2006; Bisi et al. 2008; Webb et al. 2013). With the aid of numerical modeling, coordinated imaging in WL and FR enables the properties and evolution of complex interplanetary dynamics to be diagnosed.

\section{CONCLUDING REMARKS}

In this paper, we have investigated the WL and FR signatures of an interplanetary shock based on an approach of forward MHD modeling. The WL Thomson scattering geometry is increasingly more significant at larger elongations. The degree of WL polarization can be used to estimate the 3D location of the main scattering region, while FR measurements can be used to infer, to some extent, the magnetic configurations of CMEs. This work demonstrates, as a proof of concept, that the availability of coordinated observations in polarized WL and FR measurements enables an estimate of the local LOS magnetic component. Although the current generation of heliospheric WL imagers, such as the STEREO/HI instruments, do not have polarizers, there are advances underway in terms of FR imaging using low-frequency radio arrays. Coordinated imaging in WL and FR would enable the inner heliosphere to be mapped in fine detail; the locations, masses, and magnetic fields of CMEs can be diagnosed on the basis of such combined observations. Forward modeling is crucial for establishing a causal link between interplanetary dynamics and observable signatures, and can provide valuable guidance for future coordinated WL and FR imaging.

Although numerical MHD models of the inner heliosphere were not the methodology of the current work, this technique can also be directly driven by photospheric observations (e.g., Hayashi 2005; Wu et al. 2006; Feng et al. 2012b). A comparison of synthesized and observed WL and FR sky maps, the former based on the use of such data-driven models, would prove highly beneficial for validating the forward modeling and interpreting the observations. Such an integration of observation data analysis and numerical forward modeling will be explored as a continuation of the preliminary modeling work presented in this paper.

This work is jointly supported by the National Basic Research Program (program 973) under grant 2012CB825601, the Chinese Academy of Sciences (KZZD-EW-01-4), the National Natural Science Foundation of China (41231068, 41031066, 41204129, 41374175), the Strategic Priority Research Program on Space Science from the Chinese Academy of Sciences (XDA04060801), the Specialized Research Fund for State Key Laboratories of China, the Chinese Public Science and Technology Research Funds Projects of the Ocean (201005017), the Open Research Foundation of Science and Technology on Aerospace Flight Dynamics Laboratory of China (AFDL2012002), and the research fund for recipients of the excellent award of the Chinese Academy of Sciences President's scholarship (startup fund). Ming Xiong is also partially supported by an institutional project "Key Fostering Direction in Pulsar Science and Application" from the Center of Space Science and Applied Research, China. Ming Xiong sincerely thanks Drs. Bo Li, Ding Chen, Craig DeForest, James Tappin, and Tim Howard for their helpful discussions and thoughtful suggestions. We sincerely thank the anonymous referee for constructive suggestions.

\section{REFERENCES}

Akioka, M., Nagatsuma, T., Miyake, W., Ohtaka, K., \& Marubashi, K. 2005, AdSpR, 35, 65

Bemporad, A., Zuccarello, F. P., Jacobs, C., Mierla, M., \& Poedts, S. 2012, SoPh, 281,223

Biesecker, D. A., Webb, D., \& St. Cyr, O. 2008, SSRv, 136, 45

Billings, D. E. 1966, A Guide to the Solar Corona (San Diego, CA: Academic Press)

Bird, M. K., Schrüfer, E., Volland, H., \& Sieber, W. 1980, Natur, 283, 459

Bird, M. K., Volland, H., Howard, R. A., et al. 1985, SoPh, 98, 341

Bisi, M. M., Jackson, B. V., Hick, P. P., et al. 2008, JGR, 113, A00A11

Burlaga, L. F., Behannon, K. W., \& Klein, L. W. 1987, JGR, 92, 5725

Burlaga, L. F., Plunkett, S. P., \& Cyr, O. C. S. 2002, JGR, 107, 1266

Burlaga, L. F., Sittler, E., Mariani, F., \& Schwenn, R. 1981, JGR, 86, 6673

Cargill, P. J. 2004, SoPh, 221, 135

Cargill, P. J., Chen, J., Spicer, D. S., \& Zalesak, S. T. 1996, JGR, 101, 4855

Chen, J. 1989, ApJ, 338, 453

Chen, J. 1996, JGR, 101, 27499

Chen, Y., Li, G. Q., \& Hu, Y. Q. 2006, ApJ, 649, 1093

Crifo, F., Picat, J. P., \& Cailloux, M. 1983, SoPh, 83, 143

Crooker, N. U., Huang, C. L., Lamassa, S. M., et al. 2004, JGR, 109, A03107

Davis, C. J., Davies, J. A., Lockwood, M., et al. 2009, GeoRL, 36, L08102

Davis, C. J., Kennedy, J., \& Davies, J. A. 2010, SoPh, 263, 209

Davies, J. A., Harrison, R. A., Perry, C. H., et al. 2012, ApJ, 750, 23

Davies, J. A., Harrison, R. A., Rouillard, A. P., et al. 2009, GeoRL, 36, L02102

de Koning, C. A., Pizzo, V. J., \& Biesecker, D. A. 2009, SoPh, 256, 167

de Vos, M., Gunst, A. W., \& Nijboer, R. 2009, IEEEP, 97, 1431

DeForest, C., Howard, T., \& Tappin, S. 2011, ApJ, 738, 103

DeForest, C. E., Howard, T. A., \& Tappin, S. J. 2013, ApJ, 765, 44

Démoulin, P. 2010, AIP Conf. Proc. 1216, 12th International Solar Wind Conference, ed. M. Maksimovic, K. Issautier, N. Meyer-Vernet, M. Moncuquet, \& F. Pantellini (Melville, NY: AIP), 329

Démoulin, P., \& Dasso, S. 2009, A\&A, 498, 551

Démoulin, P., Dasso, S., \& Janvier, M. 2013, A\&A, 550, A3

Echer, E., Alves, M. V., \& Gonzalez, W. D. 2004, SoPh, 221, 361

Eyles, C. J., Harrison, R. A., Davis, C. J., et al. 2009, SoPh, 254, 387

Eyles, C. J., Simnett, G. M., Cooke, M. P., et al. 2003, SoPh, 217, 319

Farrugia, C. J., Burlaga, L. F., Osherovich, V. A., et al. 1993, JGR, 98, 7621

Feng, L., Inhester, B., Wei, Y., et al. 2012a, ApJ, 751, 18

Feng, X., Jiang, C., Xiang, C., Zhao, X., \& Wu, S. T. 2012b, ApJ, 758, 62

Goldshmidt, O., \& Rephaeli, Y. 1993, ApJ, 411, 518

Gómez, J., Marscher, A. P., Jorstad, S. G., Agudo, I., \& Roca-Sogorb, M. 2008, ApJL, 681, L69

Gonzalez, W., Tsurutani, B., \& Gonzalez, C. D. 1999, SSRv, 88, 529

Gopalswamy, N. 2006, SSRv, 124, 145

Gopalswamy, N., Davila, J. M., St. Cyr, O. C., et al. 2011, JASTP, 73, 658

Gopalswamy, N., Yashiro, S., Kaiser, M. L., Howard, R. A., \& Bougeret, J. L. 2001, ApJL, 548, L91

Gosling, J. T., \& McComas, D. J. 1987, GeoRL, 14, 355

Gosling, J. T., \& Pizzo, V. J. 1999, SSRv, 89, 21

Groth, C. P. T., De Zeeuw, D. L., Gombosi, T. I., \& Powell, K. G. 2000, JGR, 105,25053

Gulisano, A. M., Démoulin, P., Dasso, S., \& Rodriguez, L. 2012, A\&A, 543, A107

Gulisano, A. M., Démoulin, P., Dasso, S., Ruiz, M. E., \& Marsch, E. 2010, A\&A, 509, A39

Harrison, R. A., Davis, C. J., Eyles, C. J., et al. 2008, SoPh, 247, 171

Harrison, R. A., Davies, J. A., Möstl, C., et al. 2012, ApJ, 750, 45

Harrison, R. A., Davies, J. A., \& Rouillard, A. P. 2009, SoPh, 256, 219

Hayashi, K. 2005, ApJS, 161, 480

Hollweg, J. V., Cranmer, S. R., \& Chandran, B. D. G. 2010, ApJ, 722, 1495

Howard, T. A., \& DeForest, C. E. 2012, ApJ, 752, 130

Howard, T. A., \& Tappin, S. J. 2009, SSRv, 147, 31

Howard, T. A., Tappin, S. J., Odstrcil, D., \& DeForest, C. E. 2013, ApJ, 765, 45 Hu, Q., \& Sonnerup, B. U. Ö. 2002, JGR, 107, 1142

Hu, Y. Q., \& Jia, X. Z. 2001, JGR, 106, 29,299

Jackson, B., Buffington, A., Hick, P., Bisi, M., \& Clover, J. 2010, SoPh, 265,257

Jackson, B. V., Buffington, A., Hick, P. P., Wang, X., \& Webb, D. F. 2006, JGR, 111, A04S91

Jensen, E. A. 2007, PhD dissertation, Univ. California, Los Angeles, USA

Jensen, E. A., Hick, P. P., Bisi, M. M., et al. 2010, SoPh, 265, 31

Jensen, E. A., Nolan, M., Bisi, M. M., Chashei, I., \& Vilas, F. 2013, SoPh, 285,71

Jensen, E. A., \& Russell, C. T. 2008, GeoRL, 35, L02103

Jokipii, J. R., \& Lerche, I. 1969, ApJ, 157, 1137 
Klein, L. W., \& Burlaga, L. F. 1982, JGR, 87, 613

Leinert, C., \& Pitz, E. 1989, A\&A, 210, 399

Lepping, R. P., Burlaga, L. F., \& Jones, J. A. 1990, JGR, 95, 11957

Levy, G., Sato, T., Seidel, B. L., et al. 1969, Sci, 166, 596

Li, B., \& Li, X. 2008, ApJ, 682, 667

Li, B., Li, X., \& Labrosse, N. 2006, JGR, 111, A08106

Liu, Y., Davies, J. A., Luhmann, J. G., et al. 2010, ApJL, 710, L82

Liu, Y., Luhmann, J. G., Lugaz, N., et al. 2013, ApJ, 769, 45

Liu, Y., Luhmann, J. G., Möstl, C., et al. 2012, ApJL, 746, L15

Liu, Y., Manchester, W. B., IV, Kasper, J. C., Richardson, J. D., \& Belcher, J. W. 2007, ApJ, 665, 1439

Liu, Y., Manchester, W. B., IV., Richardson, J. D., et al. 2008, JGR, 113, A00B03

Lonsdale, C. J., Cappallo, R. J., Morales, M. F., et al. 2009, IEEEP, 97, 1497

Lugaz, N., Farrugia, C. J., Davies, J. A., et al. 2012, ApJ, 759, 68

Lugaz, N., Hernandez-Charpak, J. N., Roussev, I. I., et al. 2010, ApJ, 715, 493

Lugaz, N., \& Kintner, P. 2013, SoPh, 285, 281

Lugaz, N., Manchester, W. B. IV, \& Gombosi, T. I. 2005, ApJ, 634, 651

McComas, D. J., Barraclough, B., Funsten, H., et al. 2000, JGR, 105, 10419

Moran, T. G., \& Davila, J. M. 2004, Sci, 305, 66

Moran, T. G., Davila, J. M., \& Thompson, W. T. 2010, ApJ, 712, 453

Möstl, C., \& Davies, J. A. 2013, SoPh, 285, 411

Möstl, C., Farrugia, C. J., Kilpua, E. K. J., et al. 2012, ApJ, 758, 10

Möstl, C., Rollett, T., Lugaz, N., et al. 2011, ApJ, 741, 34

Nakwacki, M. S., Dasso, S., Démoulin, P., Mandrini, C. H., \& Gulisano, A. M. 2011, A\&A, 535, A52

Oberoi, D., \& Lonsdale, C. J. 2012, RaSc, 47, RS0K08

Odstrčil, D., \& Pizzo, V. J. 2009, SoPh, 259, 297

Odstrčil, D., Smith, Z., \& Dryer, M. 1996, GeoRL, 23, 2521

Oliveros, J. C. M., Raftery, C. L., Bain, H. M., et al. 2012, ApJ, 748, 66

Owens, M., Cargill, P., Pagel, C., Siscoe, G., \& Crooker, N. 2005, JGR, 110, A01105

Parker, E. N. 1958, ApJ, 128, 664

Pätzold, M., \& Bird, M. K. 1998, GeoRL, 25, 2105

Pizzo, V. J., \& Biesecker, D. A. 2004, GeoRL, 31, L21802

Plunkett, S. P., Thompson, B. J., Howard, R. A., et al. 1998, GeoRL, 25, 2477

Poland, A. I., \& Munro, R. H. 1976, ApJ, 209, 927

Reichstein, A., \& Gabuzda, D. 2012, JPhCS, 355, 012021

Rouillard, A. P., Davies, J. A., Forsyth, R. J., et al. 2008, GeoRL, 35, L10110

Rouillard, A. P., Davies, J. A., Forsyth, R. J., et al. 2009a, JGR, 114, A07106

Rouillard, A. P., Savani, N. P., Davies, J. A., et al. 2009b, SoPh, 256, 307
Sakurai, T., \& Spangler, S. R. 1994, ApJ, 434, 773

Savani, N. P., Davies, J. A., Davis, C. J., et al. 2012, SoPh, 279, 517

Savani, N. P., Owens, M. J., Rouillard, A. P., Forsyth, R. J., \& Davies, J. A. 2010, ApJL, 714, L128

Sheeley, N. R., Jr., Herbst, A. D., Palatchi, C. A., et al. 2008, ApJ, 674, 109

Shen, C., Wang, Y., Wang, S., et al. 2012a, NatPh, 8, 923

Shen, F., Wu, S. T., Feng, X. S., \& Wu, C. C. 2012 b, JGR, 117, A11101

Steed, K., Owen, C. J., Démoulin, P., \& Dasso, S. 2011, JGR, 116, A01106

Szajko, N., Cristiani, G., Mandrini, C., \& Dal Lago, A. 2013, AdSpR, 51, 1842

Tappin, S. J., Buffington, A., Cooke, M. P., et al. 2004, GeoRL, 31, L02802

Temmer, M., Rollett, T., Möstl, C., et al. 2011, ApJ, 743, 101

Temmer, M., Vršnak, B., Rollett, T., et al. 2012, ApJ, 749, 57

Thernisien, A., Vourlidas, A., \& Howard, R. A. 2009, SoPh, 256, 111

Thompson, A. R., Clark, B. G., Wade, C. M., \& Napier, P. J. 1980, ApJS, 44,151

Thompson, B. J., Plunkett, S. P., Gurman, J. B., et al. 1998, GeoRL, 25, 2465

Tsurutani, B. T., Lee, Y. T., Gonzalez, W. D., \& Tang, F. 1992, GeoRL, 19, 73

Tsurutani, B. T., Smith, E. J., Gonzalez, W. D., Tang, F., \& Akasofu, S. I. 1988, JGR, 93, 8519

Vourlidas, A., \& Howard, R. A. 2006, ApJ, 642, 1216

Vršnak, B., \& Gopalswamy, N. 2002, JGR, 107, 1019

Wang, Y. M., Ye, P. Z., \& Wang, S. 2003, JGR, 108, 1370

Webb, D. F., \& Howard, T. A. 2012, LRSP, 9, 3

Webb, D. F., Möstl, C., Jackson, B. V., et al. 2013, SoPh, 285, 317

Winterhalter, D., Smith, E. J., Burton, M. E., Murphy, N., \& McComas, D. J. 1994, JGR, 99, 6667

Woo, R. 1997, GeoRL, 24, 97

Wu, S. T., Wang, A. H., Liu, Y., \& Hoeksema, J. T. 2006, ApJ, 652, 800

Xiong, M., Breen, A., Bisi, M., et al. 2011, JASTP, 73, 1270

Xiong, M., Davies, J. A., Bisi, M. M., et al. 2013, SoPh, 285, 369

Xiong, M., Zheng, H. N., \& Wang, S. 2009, JGR, 114, A11101

Xiong, M., Zheng, H. N., Wang, Y. M., \& Wang, S. 2006a, JGR, 111, A08105

Xiong, M., Zheng, H. N., Wang, Y. M., \& Wang, S. 2006b, JGR, 111, A11102

Xiong, M., Zheng, H. N., Wu, S. T., Wang, Y. M., \& Wang, S. 2007, JGR, 112 A11103

You, X. P., Coles, W. A., Hobbs, G. B., \& Manchester, R. N. 2012, MNRAS, 422, 1160

Zavala, R. T., \& Taylor, G. B. 2002, ApJL, 566, L9

Zhang, J., Richardson, I. G., \& Webb, D. F. 2008, JGR, 113, A00A12

Zhang, Y., Du, A. M., Feng, X., et al. 2013, SoPh, in press 